\title{
Valoración de un seguro de vida mediante opciones exóticas
}

\author{
PESCE, GABRIELA \\ Universidad Nacional del Sur (Argentina) \\ Correo electrónico: gabriela.pesce@uns.edu.ar \\ MiLANESI, GASTÓN \\ Universidad Nacional del Sur (Argentina) \\ Correo electrónico: milanesi@uns.edu.ar \\ El AlABi, EMILIO \\ Universidad Nacional del Sur (Argentina) \\ Correo electrónico: emilio.elalabi@uns.edu.ar \\ MENNA, JOAQUÍN \\ Universidad Nacional del Sur (Argentina) \\ Correo electrónico: joaquin.menna@hotmail.com
}

\begin{abstract}
RESUMEN
Este trabajo presenta el análisis y valoración de seguros de vida individual, temporarios y con prima nivelada, a partir de la analogía de las reglas del contrato con las de una opción exótica, en particular una digital pura o pulso, también conocida como cash or nothing. Se presentan diversos casos que parten de un asegurado con atributos cambiantes (edad y género) y se testea la sensibilidad a diferentes formas funcionales para la distribución de probabilidad de la variable estocástica, tiempo de vida restante al momento de contratar el seguro, mediante simulaciones de Monte Carlo. En un conjunto de casos la función de probabilidad se ajusta de manera personalizada a datos recientes de la República Argentina para estimar las probabilidades de ejercicio de la opción, variable que resulta sensiblemente crítica para la estimación del valor del contrato. Los valores de mercado de las primas de las pólizas comparables superan en más del doble al valor teórico encontrado para la opción exótica, ante iguales condiciones del contrato en cuanto a monto asegurado, duración y condiciones demográficas del individuo.
\end{abstract}

Palabras clave: opción exótica, seguro de vida, digital pura, probabilidad de ejercicio.

Clasificación JEL: G13; G22; C15.

MSC2010: 91B30; 91G20; 97M30. 


\title{
Life insurance valuation using exotic options
}

\begin{abstract}
This paper presents the analysis and valuation of an individual, temporary, and leveled-prime life insurance. It starting point is an analogy between contract rules and a financial exotic option. In particular, a cash or nothing option. Several cases are presented from a person with different age and gender, and sensitivity to different probability distributions are tested using Monte Carlo simulation. All cases are adjusted to Argentinean recent data in order to estimate exercise prices, main variable to estimate the contract value. Prime market values used on this work are more than double than the theoretical value found on the exotic option while comparing them to identical contract conditions such as insured amount, time frame and demographic conditions of the individual.
\end{abstract}

Keywords: exotic option, life insurance, digital option, exercise probability.

JEL classification: G13; G22; C15.

MSC2010: 91B30; 91G20; 97M30.

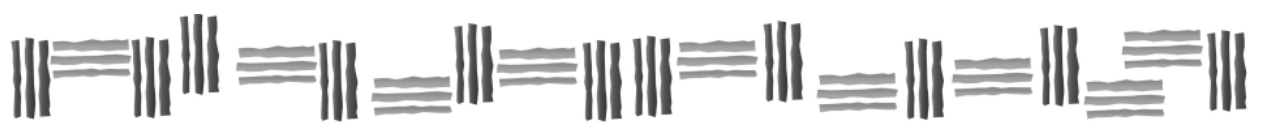




\section{Introducción.}

La teoría financiera provee de un conjunto de instrumentos y modelos que contribuyen a valorar activos en situaciones contingentes y que, por analogía, pueden ser aplicados a la valoración, medición y cobertura de riesgos sobre activos reales. Sin embargo, en la práctica, tales herramientas son empleadas con muy poca frecuencia, siendo mayoritariamente utilizados los métodos tradicionales, que poco explican sobre algunas situaciones controvertidas de determinados activos no financieros. En particular, este trabajo se desarrolla en el marco de un proyecto de investigación que tiene como objetivo aplicar la teoría de derivados exóticos a problemas complejos, en este caso, la incertidumbre ante los años de vida restantes del tomador de la opción como subyacente del contrato de seguro.

El artículo presenta el análisis y valoración de seguros de vida individual, temporarios y con prima nivelada, a partir de la analogía de las reglas del contrato y matrices de pago con las de una opción exótica, en particular una digital pura, también conocida como cash or nothing, como alternativa a los modelos actuariales. El trabajo utiliza datos actuales de la República Argentina para estimar las probabilidades de ejercicio de la opción, variable que resulta sensiblemente crítica para la estimación del valor del contrato.

El manuscrito se estructura presentando en la segunda sección el marco conceptual, que resume algunos antecedentes del uso de modelos financieros para la valoración de seguros, lo que resulta fundamental para la presentación teórica sobre opciones exóticas, particularmente sobre las binarias y su valoración, tipología que atañe en este estudio. Luego se detalla brevemente el apartado metodológico, para continuar, en la cuarta sección, con el análisis del estudio de caso, caracterizando las principales reglas de los seguros de vida individual, de qué depende su valor y describiendo la probabilidad de ejercicio de la opción, asociada a la muerte del asegurado. En el quinto apartado se presentan los resultados de la valoración del contrato, describiendo la anatomía del valor intrínseco y valorando a partir de un ajuste sobre modelo propuesto por Black and Scholes (1973), partiendo de casos sencillos y presentando extensiones que cambian algunos supuestos que generan resultados más reales y más complejos, que son comparados con los valores de mercado. Finalmente, en la quinta sección se presentan las conclusiones y futuras líneas de trabajo.

\section{Marco teórico.}

\subsection{Antecedentes del uso de modelos financieros para valorar seguros.}

Existen numerosos trabajos que incorporan el uso de modelos financieros para valorar distintos tipos de seguros. Inicialmente, D'Arcy y Doherty (1988) proponen la idea que una compañía de seguros es similar a cualquier empresa con la particularidad que su pasivo está compuesto por el conjunto de las pólizas emitidas. A lo largo de su libro, utilizan los modelos financieros tradicionales para valorar seguros de hogar partiendo de algunas distinciones entre estos modelos y los modelos actuariales convencionales. Dentro de ellos se puede mencionar que se asume que los asegurados son neutrales al riesgo y que las aseguradoras no tienen funciones de utilidad; que los precios son determinados por la oferta y demanda de mercados eficientes de forma semifuerte; y que el valor del dinero en el tiempo es explícitamente conocido. Los autores describen las fortalezas y las debilidades de los modelos financieros tradicionales utilizados para valorar estos seguros, como son el modelo de valoración de activos de capital (Capital Asset Pricing Model [CAPM]), el modelo de fijación de precios por arbitraje (Arbitrage Pricing Theory [APT]), modelos de descuento de flujo de fondos y modelos de valoración de opciones.

Con argumentos similares, Cummins (1990) también propone la utilización de modelos financieros para la valoración de seguros. Critica los modelos actuariales tradicionales manifestando que, al igual que D'Arcy y Doherty (1988), estos modelos solo consideran la oferta en la preciación del seguro, sin tener en cuenta la demanda. En un trabajo un año más tarde, Cummins (1991) critica la 
estadística utilizada por los modelos actuariales convencionales y justifica nuevamente la importancia de la inclusión de los modelos financieros tradicionales en la valoración de seguros.

Del Pozo (2001) presenta un trabajo en el que compara la valoración de seguros a través de modelos financieros tradicionales como CAPM y APT y a través de los métodos de valoración de opciones. Concluye que utilizando los métodos de valoración de opciones como puede ser el modelo de Black-Scholes-Merton (Black \& Scholes, 1973; Merton, 1973), evita los problemas de estimación de variables que tienen los modelos como CAPM y APT. Argumenta que en los modelos de opciones no se tienen que estimar variables de riesgo de las primas ya que están implícitas en el activo subyacente de la opción. Sin embargo, la variable estocástica debe seguir una distribución normal o log-normal.

Finalmente, Gatzert y Schmeiser (2006) así como Luoma, Puustelli y Koskinen (2008) proponen la valoración de seguros de vida utilizando modelos de valoración de opciones. Sin embargo, declaran que los modelos clásicos tienen grandes dificultades para poder incorporar el comportamiento de los tenedores del contrato en donde los mismos tienen numerosas opciones implícitas en sus cláusulas (Gatzert \& Schmeiser, 2006). Por ejemplo, existen contratos que tienen un mínimo interés asegurado, un interés anual o al terminar el contrato que puede ser asegurado o no, una opción de rendición para poder vender su opción al lanzador a un precio estipulado previamente, una opción paid-up que permite dejar de pagar las primas una vez que haya acumulado cierta cantidad de dinero en su cuenta, entre otras. Este tipo de antecedentes da lugar a la aplicación de opciones exóticas en pos de mejorar las estimaciones de algunas primas de contratos de seguros.

\subsection{Opciones exóticas.}

Los derivados exóticos, no usuales o no estándares presentan un cambio en la matriz de pagos, sus reglas de ejercicio y sus características de valor (Kolb, 2003; Whaley, 2006; Gaarder, 2007; Wilmott, 2009; Kolb \& Overdahl, 2010; Hull, 2012). Si bien en la mayoría de los libros de textos se discute un conjunto acotado de este tipo de derivados, en el mercado se observa un número ilimitado de variaciones de estos tipos de contratos. Algunos pueden valorarse de manera analítica y otros mediante procedimientos numéricos, al no existir solución cerrada para los mismos. Es importante pensar en sus potenciales aplicaciones, ya que en algunos casos el contrato es un elemento de gestión del riesgo, dadas su sensibilidad y capacidad de replicar riesgos y rendimientos. En otras ocasiones surge con el objetivo de convertirse en una apuesta especulativa. Finalmente, las opciones exóticas pueden replicar situaciones complejas, con la finalidad de predecir probabilidades de ocurrencia o valorar los efectos económicos de situaciones contingentes (probabilidades de fracasos financieros, valoración de externalidades, riesgos ambientales y contingencias personales). La Figura 1 detalla un conjunto limitado de opciones exóticas clásicas comúnmente desarrolladas en los textos especializados (Fernández \& Ariño, 1996; García et al., 2000; Casparri \& García, 2010).

A los fines del presente trabajo son de especial interés las opciones exóticas del tipo "todo o nada" u opciones digitales pura. En tal sentido se pretende valorar los seguros de vida individuales, habida cuenta la simetría que existe entre las disposiciones contractuales de la cobertura de vida y el flujo al vencimiento en este tipo de opciones. Dentro este conjunto se distinguen las opciones binarias conocidas como "activo o nada" (asset-or-nothing), donde el tenedor recibe el activo en caso de estar dentro del dinero, y las digitales puras o pulso, conocidas como "dinero o nada" (cash or nothing), que son opciones binarias con un pago total o nulo en función a la ocurrencia del evento. 
Figura 1. Opciones exóticas clásicas.

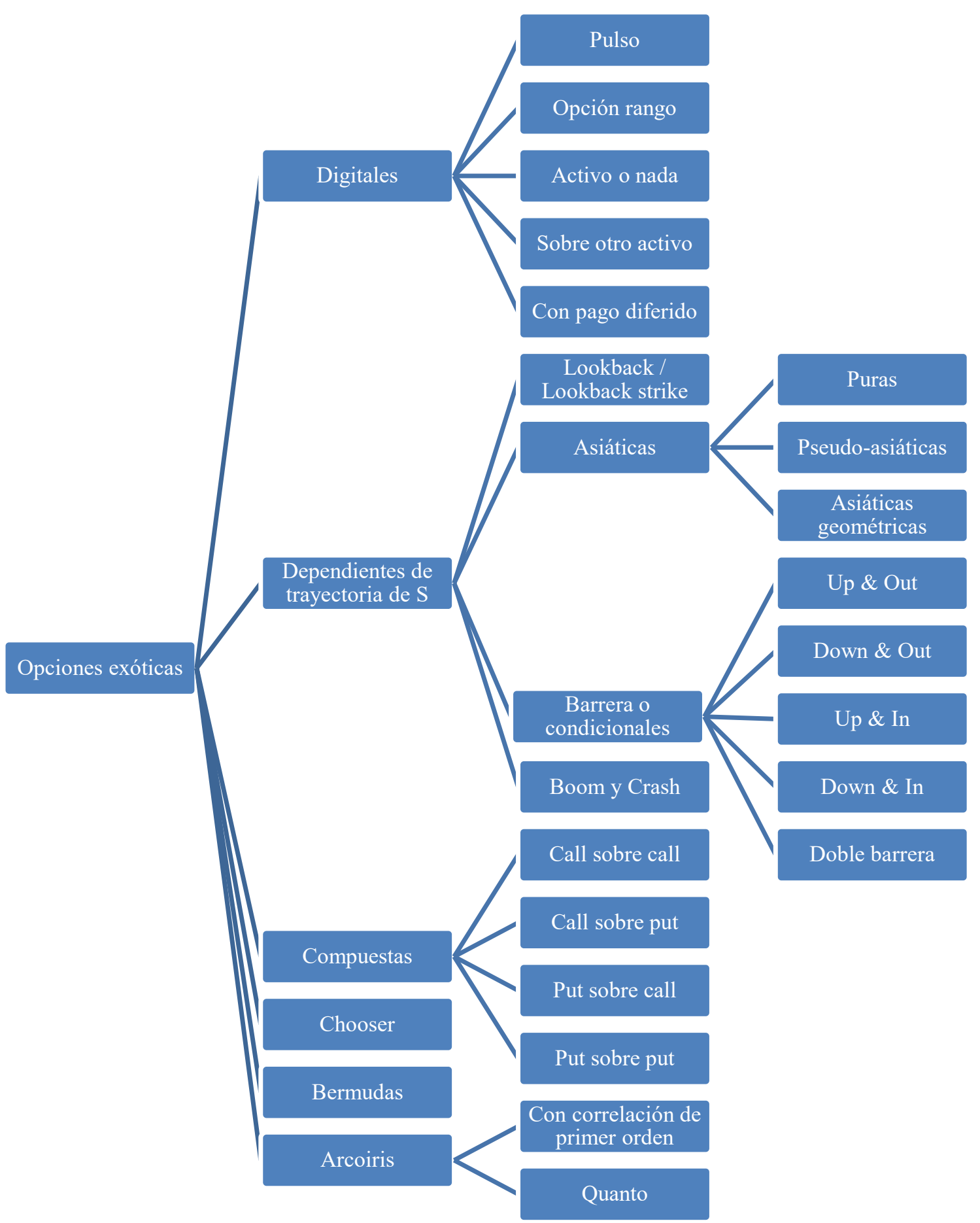

Fuente: Elaboración propia.

\subsection{Valoración de opciones exóticas del tipo todo o nada.}

Una opción de compra del tipo "activo o nada" paga una unidad del activo subyacente al momento $T$ siempre que el precio del activo supere el precio de ejercicio. En la siguiente figura se presenta el resultado terminal correspondiente a este tipo de opción. 
Figura 2. Perfil de beneficios al vencimiento opción de compra "activo o nada".

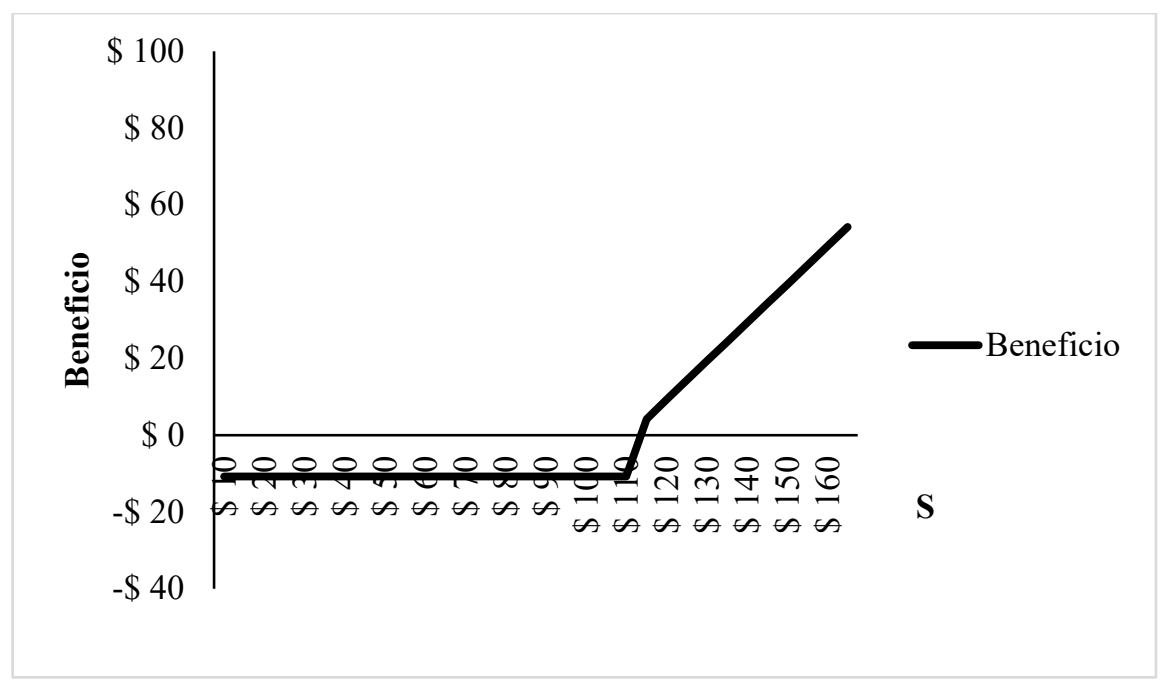

Fuente: Elaboración propia.

Para valores del activo por debajo del precio de ejercicio el titular de la opción pierde el valor de la prima pagada. Para valores superiores al precio de ejercicio, el propietario de la opción recibe la unidad del activo, cubriendo el costo de la prima. Para una unidad monetaria de activo bajo los supuestos del modelo Black-Scholes-Merton o BSM (Black \& Scholes, 1973; Merton, 1973), el valor teórico de una opción de este tipo es (Ecuaciones 1, 2 y 3):

$$
c_{A O N}=e^{-i T} N\left(d_{1}\right)
$$

donde:

$$
d_{1}=\frac{\ln \left(S_{T} e^{-i T} / X e^{-r T}\right)+0.5 \sigma^{2} T}{\sigma \sqrt{T}}
$$

$\mathrm{y}$

$$
d_{2}=d_{1}-\sigma \sqrt{T}
$$

siendo $S_{T}$ el valor esperado del activo, $i$ la tasa de rendimiento del activo (dividend yield), $X$ el precio de ejercicio, $r$ el tipo de interés libre de riesgo, $\sigma$ la volatilidad del precio del activo subyacente, $T$ el tiempo al vencimiento, $\mathrm{d}_{1} \mathrm{y} \mathrm{d}_{2}$ los parámetros de la distribución de probabilidad que permiten calcular $\mathrm{N}\left(\mathrm{d}_{1}\right)$, que se interpreta como la probabilidad de ejercicio de la opción.

El valor terminal de una opción de compra "dinero o nada" se expone en la siguiente figura. 
Figura 3. Perfil de beneficios al vencimiento opción de compra "dinero o nada".

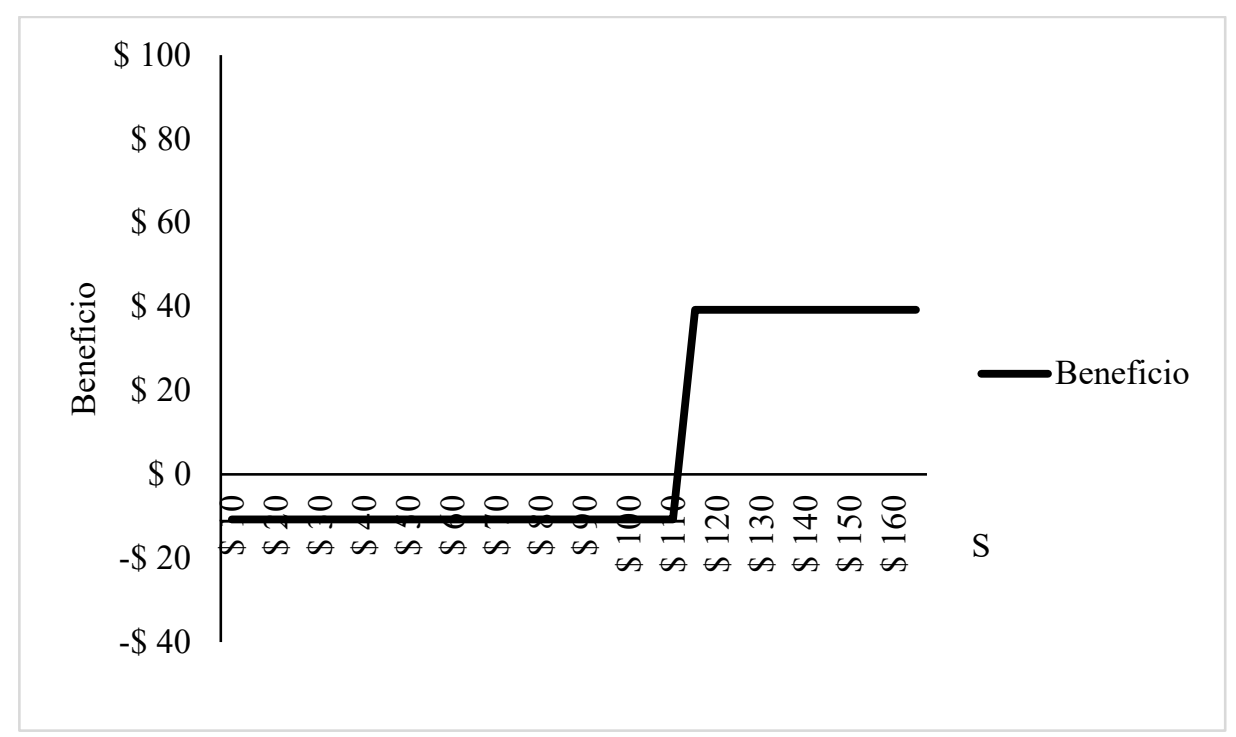

Fuente: Elaboración propia.

Para valores por debajo del precio de ejercicio el poseedor de la opción pierde la prima, mientras que para valores superiores recibe una unidad monetaria. Igual que en el caso precedente, suponiendo $\mathrm{BSM}$, el valor de esta opción es la siguiente:

$$
c_{\text {CON }}=e^{-r T} N\left(d_{2}\right)
$$

donde el valor fijo a recibir en la Ecuación 4 equivale a una unidad monetaria. Una opción de compra europea otorga el derecho de adquirir el subyacente por un valor igual al precio de ejercicio $X$, a fecha de vencimiento $T$. La cartera réplica de los flujos correspondientes a una opción de compra consiste en comprar $S$ unidades de una opción de compra del tipo "activo o nada" y vender $X$ unidades de una opción de compra del tipo "dinero o nada". El valor de una opción de compra europea es la diferencia entre una posición larga de una opción de compra "activo o nada" y una posición corta en una opción de compra "dinero o nada" (Ecuación 5).

$$
c_{\mathrm{BSM}}=c_{\mathrm{AON}}-c_{\mathrm{CON}}=S e^{-i T} N\left(d_{1}\right)-X e^{-r T} N\left(d_{2}\right)
$$

En el caso de una opción de venta del tipo "activo o nada", para una unidad de activo su valor es la siguiente:

$$
p_{A O N}=e^{-i T} N\left(-d_{1}\right)
$$

La expresión para valorar una opción de venta del tipo "dinero o nada" sobre una unidad monetaria es como se muestra a continuación:

$$
p_{C O N}=e^{-r T} N\left(-d_{2}\right)
$$

Una opción de venta europea otorga el derecho de vender el subyacente por un valor igual al precio de ejercicio $X$, a fecha de vencimiento $T$. La cartera réplica de los flujos correspondientes a una opción de venta consiste en vender $S$ unidades de una opción de venta del tipo "activo o nada" y comprar $X$ unidades de una opción de venta del tipo "dinero o nada". El valor de una opción de venta europea es la diferencia entre una posición corta de una opción de venta "activo o nada" y una posición larga en una opción de venta "dinero o nada" (Ecuación 8): 


$$
p_{\mathrm{BSM}}=p_{\mathrm{CON}}-p_{A O N}=X e^{-r T} N\left(-d_{2}\right)-S e^{-i T} N\left(-d_{1}\right)
$$

En el caso de las opciones todo o nada se presentan ciertas características distintivas en relación al riesgo. En particular respecto de la distribución del valor del coeficiente delta (sensibilidad o derivada primera del valor de la opción en relación a las variaciones en el precio del activo subyacente), para opciones que se encuentran significativamente fuera del dinero, el valor del delta es cercano a cero. La causa de este valor es lógica. Si prácticamente no existen posibilidades (en términos de distribución normal) de que la opción se encuentre en el dinero al vencimiento, la opción de compra del tipo "activo o nada" es insensible a pequeños movimientos en el valor del activo. Por el contrario, cuando este tipo de opción se encuentra profundamente en el dinero, presenta valores de delta cercanos a uno. La opción tiende a valer lo mismo que el subyacente. Asimismo, y a diferencia de las opciones europeas tradicionales, el valor del delta de una opción de compra del tipo "activo o nada" puede superar el valor de uno e incrementarse cuando se encuentra fuertemente dentro del dinero y su vencimiento es cercano. El valor máximo del delta es igual al valor del activo. Ante el inminente vencimiento, frente a un movimiento en el precio del activo, para aquellas opciones levemente fuera y en el dinero, el valor de la opción de compra "activo o nada" va de 0 a S.

\section{Metodología.}

El trabajo se aborda mediante un estudio de caso simulado, bajo la forma de un estudio de caso único con unidades incorporadas (Yin, 2005), a partir del análisis de diferentes contratos de seguros de vida individuales.

La elección del caso analizado fue realizada teniendo en cuenta el juicio de los investigadores, buscando contratos con subyacentes no financieros, donde la teoría de opciones exóticas pueda aportar un análisis complementario al realizado desde el punto de vista actuarial. El enfoque utilizado para analizar el caso es teórico empírico, el abordaje es cuantitativo y el alcance de la investigación es descriptivo.

Para la presentación del estudio de caso, se trabaja con información secundaria proveniente de una revisión documental, a partir de siete pólizas de cuatro compañías aseguradoras autorizadas por la Superintendencia de Seguros de la Nación; observación directa y otras fuentes secundarias, principalmente de organismos oficiales como la Superintendencia de Seguros de la Nación (SSN), el Ministerio de Salud de la Nación, la Organización Mundial de la Salud, el Ministerio de Salud y Desarrollo Social de la Nación, entre otros. En particular, la información específica de las pólizas analizadas no se publica por cuestiones de confidencialidad de los datos provistos.

A partir del estudio de caso, se realiza un conjunto de valoraciones de una opción digital pura como prima de un contrato de seguro de vida individual. Para ello se utiliza una adaptación del modelo de BSM, trabajando con valores terminales de la opción, habida cuenta de que la distribución de probabilidad del valor del activo subyacente no es lognormal. En aras de esta adaptación se recurre a la estimación de la probabilidad de ejercicio del modelo mencionado mediante simulaciones de Monte Carlo, siendo el tiempo restante de vida del asegurado la variable estocástica, para la que se asumen diferentes patrones de probabilidad, algunos de ellos a partir de datos estadísticos reales de la República Argentina, correspondientes al anuario del 2017 del Ministerio de Salud y Desarrollo Social de la Nación.

Al momento de realizar las simulaciones, se utiliza el software Oracle Crystal Ball como complemento de Microsoft Excel ${ }^{\circledR}$. Se realizan 1.000.000 de iteraciones, asumiendo un nivel de confianza de $95 \%$. 


\section{Caso de estudio.}

\subsection{El seguro de vida.}

De acuerdo a la Superintendencia de Seguros de la Nación (SSN) de Argentina, un contrato de seguro es "un acuerdo por el cual el asegurador se obliga, mediante el pago de una prima, a resarcir un daño o cumplir la prestación convenida si ocurre el evento previsto" (Ley No 17.418, 1967, artículo 1). En particular, según la Ley Nacional de Seguros 17.418, un seguro de vida es aquel seguro de tipo personal en el que el evento previsto es generalmente el fallecimiento del asegurado, pudiéndose celebrar sobre la vida del propio contratante o la de un tercero.

En este tipo de seguro, el asegurador se obliga a pagar a los beneficiarios el capital asegurado por fallecimiento de la persona, si se produce con anterioridad a la fecha de vencimiento del contrato y de acuerdo a las condiciones de la póliza. El asegurado, que debe ser mayor de 14 y menor de 60 años, puede garantizar como riesgos principales sobre la vida, los de muerte, supervivencia o cualquier combinación de ambos, y como coberturas adicionales las de invalidez, accidente o cualquier otra que tenga por objeto cubrir riesgos que afecten la salud, existencia o integridad corporal. En términos generales, suelen no cubrirse riesgos asociados a casos de radiación nuclear, guerras, participación en empresas criminales o actos de terrorismo, suicidio voluntario, entre otros.

Como contraprestación, el asegurado se compromete a abonar un premio, conocido también como prima de tarifa o comercial, que es el importe total que abonará el tomador en concepto de prima pura (costo real del riesgo asumido), más una suma determinada para gastos y utilidad del asegurador, gastos especiales de emisión y administración, costos de financiación, comisión del productor e importes destinados al pago de tasas, impuestos y contribuciones que gravan al contrato. En función de la póliza, el asegurador puede estipular que dicha contraprestación se satisfaga mediante el pago del premio único, o de premios periódicos exigibles por anualidades o en fracciones de tiempo menores anticipadas. La prima pura del seguro de vida es una función del monto asegurado, el género del asegurado, de la edad que alcanza y de su categoría de riesgo, la cual depende del tipo de actividad que realice aquél. Las categorías de riesgo se asignan al momento de ingresar al seguro, cuando se requiera aumentar el capital asegurado, o bien, al existir una agravación de riesgo. El valor máximo que dichas primas pueden asumir se determina a partir de las tablas de mortalidad aprobadas por la SSN en los planes técnicos presentados por el asegurador. Las tarifas suelen expresarse como un porcentaje de capital asegurado.

En caso de fallecimiento, la indemnización depende de la opción seleccionada previamente por el tomador, que puede consistir en considerar el capital asegurado o el fondo acumulado de su cuenta individual (Tabla 1), o bien una combinación de ambos, en la forma prevista en la póliza. Asimismo, los beneficiarios de la indemnización podrán recibirla como pago único, en cuotas con intereses garantizados o renta vitalicia.

A modo de tipificación, los seguros de vida se pueden clasificar:

i. Según la cantidad de asegurados cubiertos en la póliza, pueden ser individuales o colectivos. Los primeros cubren la muerte de un solo asegurado, mientras que en los segundos se cubre, en forma independiente y en un único contrato de ese tomador, a varios individuos expuestos a similar riesgo.

ii. En relación a la duración, los seguros pueden ser de vida completa o temporarios. Los primeros cubren el riesgo de muerte del asegurado durante toda su vida, mientras que en los segundos la cobertura se otorga sólo durante un plazo determinado establecido en la póliza.

iii. En función al tipo de prima, los seguros pueden ser con prima nivelada o con prima anual renovable. En el caso de seguros con prima nivelada, ésta resulta constante y, por lo tanto, si 
bien excede el costo real del seguro en los primeros años, cuando el asegurado alcanza una mayor edad, la relación se invierte. En las pólizas con prima renovable, ésta se ajusta periódicamente en función de la edad del asegurado y la expectativa de vida.

iv. Según el riesgo asumido, se pueden distinguir entre seguros en caso de muerte, en los que el asegurador paga la indemnización al beneficiario en caso de fallecimiento del asegurado; y seguros en caso de vida, en los que la aseguradora abona el capital cuando el asegurado sobrevive a un determinado período de tiempo. Por último, existen los llamados seguros mixtos o dotales, en los que se combinan los seguros de muerte y de vida, siendo que el capital asegurado se paga a los beneficiarios en caso de fallecimiento del asegurado antes del vencimiento del contrato, o bien se le abona a este último si sobrevive a esa fecha.

Teniendo en cuenta la clasificación anterior, en este artículo se analiza un seguro de vida individual temporario, cuyo siniestro está determinado por la muerte del asegurado, con una estimación de prima nivelada.

Tabla 1. Conformación del fondo acumulado.

Cuenta Individual del Asegurado - Fondo Acumulado

(+) Saldo acumulado de las primas netas o puras, es decir, sin considerar derechos de emisión, cargos para gastos de adquisición y explotación, cargos por derechos de cobranzas, deducciones mensuales, tasas, sellados e impuestos.

(+) Intereses acreditados, incluyendo los intereses sin riesgo, calculados en base a una tasa de interés garantizada anual en torno al $2 \%$ en dólares estadounidenses, más los intereses excedentes que surgen de la tasa efectiva de rendimiento obtenida por el asegurador.

(-) Retiros parciales efectuados por el asegurado, que producen una disminución equivalente en el saldo de su cuenta individual y/o en la indemnización por fallecimiento

(=) Saldo del Fondo Acumulado

Fuente: Elaboración propia.

\subsection{La probabilidad de fallecimiento: el ejercicio de la opción.}

La ley de mortalidad fue desarrollada en primera instancia por Gompertz (1825), matemático inglés, hace 200 años. El autor propone que la probabilidad de muerte crece exponencialmente a medida que aumenta la edad de la persona, siguiendo una progresión geométrica. Si bien existían algunas tablas de estadísticas precarias, Gompertz construye las primeras tablas de mortalidad. Incluso, en algunas de ellas, logra formar algunos rangos etarios donde la probabilidad de fallecimiento puede llegar a ser analizada de manera conjunta.

Makeham (1860), matemático y además actuario, 35 años más tarde, argumenta mediante datos experimentales que la ley de mortalidad propuesta por Gompertz no se ajusta estrictamente a los datos de la realidad. Para ello, propone ajustarlo logarítmicamente haciendo que la probabilidad de muerte sea más cercana a los datos encontrados por el autor. Makeham propone las primeras tablas de mortalidad utilizadas por el gobierno para anualidades en planes de retiro por jubilación o incapacidad. Años más tarde, Breslow y Crowley (1974) demuestran que la probabilidad de supervivencia a medida que suceden los años sigue una distribución asintótica normal.

En este orden de ideas, existen numerosos trabajos empíricos que delinean el patrón de mortalidad a partir de ajustes de datos históricos. En esa línea de estudios, la Figura 4 presenta la probabilidad de muerte de una persona a diferentes edades, con estimaciones de datos de la población 
de Estados Unidos para el año 2003. Como puede observarse, la tasa de mortalidad crece exponencialmente a partir de edades superiores a 30 años.

Figura 4. Probabilidad de muerte anual según edad.

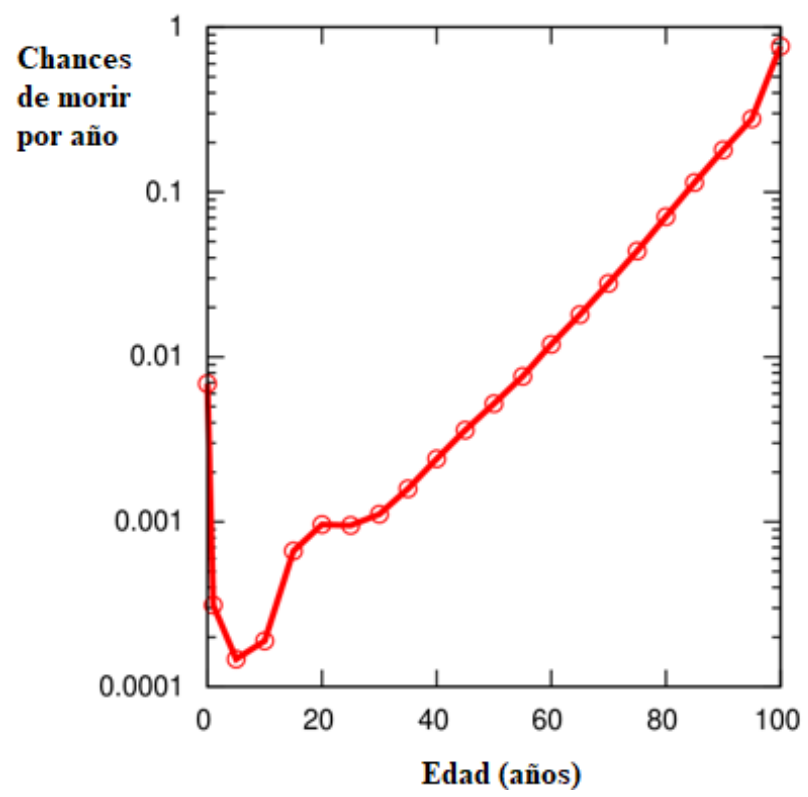

Fuente: Adaptado de National Vital Statistics Report (2006).

En cuanto a las estadísticas oficiales, en la Tabla A.1 del Apéndice se presentan las tasas de mortalidad según la Commisioner Standard Ordinary (CSO por sus siglas en inglés, 2001). De acuerdo a la información provista por el Boletín Oficial, Resolución 697/2018 "las aseguradoras deberán utilizar la Tabla de Mortalidad CSO 2001 al setenta y cuatro por ciento (74\%)" (p. 63). La estimación es realizada por edad y está desagregada entre hombres y mujeres, subdividiéndose, a su vez, entre fumadores y no fumadores.

\section{Aplicación empírica y resultados.}

Teniendo en cuenta las características descriptas para el estudio de caso presentado, se resuelve proceder con la valoración de un contrato de vida individual, temporario, cuya siniestralidad está representada por la muerte del asegurado, con una estimación de prima nivelada, es decir, constante durante la vigencia del contrato.

Para ello, en primer lugar, se identifica que dicho contrato se asimila a una opción exótica de tipo digital pura, también conocida como cash or nothing, que son opciones binarias con un pago total o nulo en función a la ocurrencia del evento. Esto es, si el tomador del seguro fallece durante la vida del contrato, su beneficiario cobra el monto asegurado, y caso contrario, no hay pago alguno.

Por las reglas del contrato se identifica una opción tipo put, dado que al producirse el siniestro el beneficiario de la opción cobra el pago fijo. El activo subyacente es el tiempo restante de vida, variable estocástica que determina el siniestro que da lugar al pago: si el tiempo restante de vida es menor al plazo del contrato del seguro de vida, la opción se encuentra dentro del dinero, siendo su valor intrínseco Q; mientras que si el tiempo restante de vida supera el plazo del contrato, la opción se encuentra fuera del dinero, siendo el valor intrínseco nulo. De esta forma, el valor intrínseco o terminal es una función discontinua, tal como se exhibe en Ecuación 9 y Gráfico 1, asumiendo un plazo de cobertura de la póliza de 10 años. 


$$
V I=\left\{\begin{array}{l}
Q \text { si } t<T \\
0 \text { si } t>T
\end{array}\right\}
$$
contrato.

siendo $V I$ el valor intrínseco, $Q$ el pago fijo, $t$ el tiempo restante de vida y $T$ el vencimiento del

Gráfico 1. Valor intrínseco de un put digital puro.

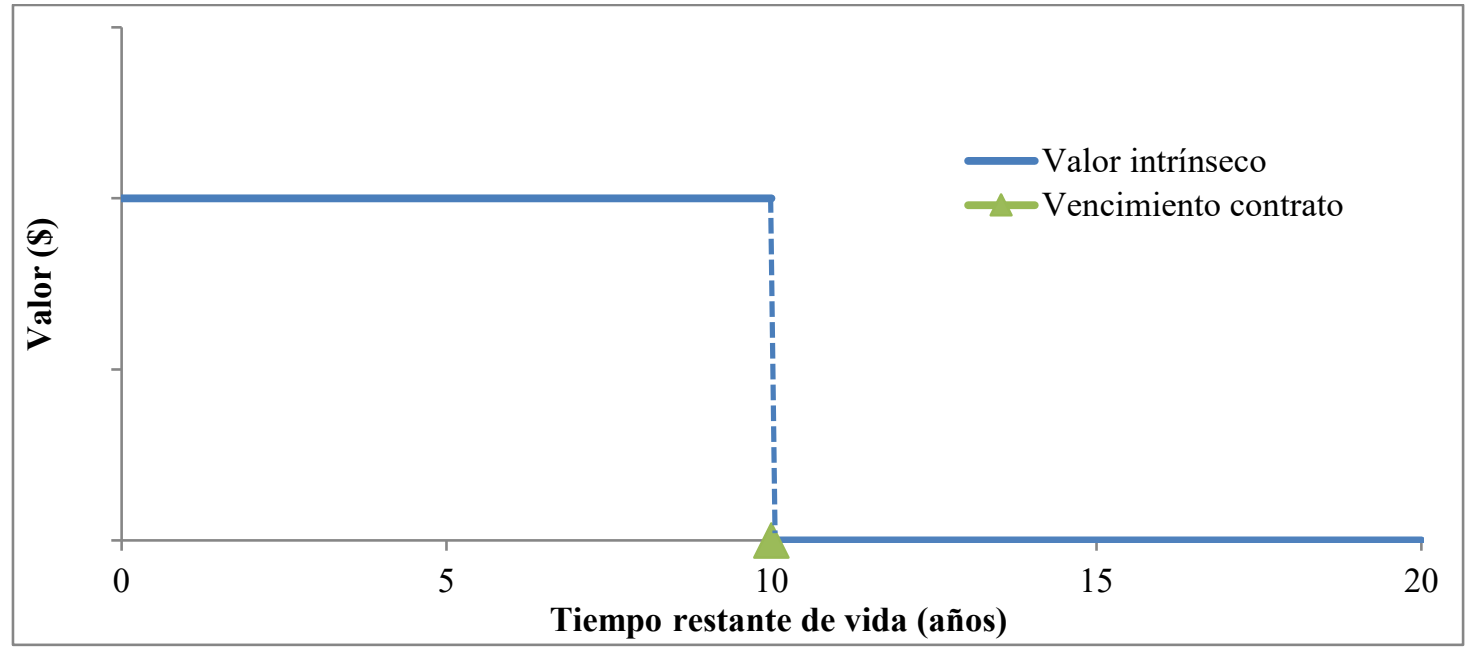

Fuente: Elaboración propia.

Como se observa en la Tabla 2, se considera un seguro con los siguientes parámetros y su analogía con la opción exótica que lo replica.

Tabla 2. Analogía entre los contratos y definición de parámetros del modelo a estimar.

\begin{tabular}{ll}
\multicolumn{1}{c}{ Seguro de vida } & \multicolumn{1}{c}{ Opción exótica digital pura } \\
\hline Prima pura de la póliza & Valor de la opción exótica digital pura \\
\hline Esperanza de vida & $\begin{array}{l}\text { Precio del activo subyacente }(\mathrm{S}) \text { : variable estocástica. } \\
\text { En la opción exótica digital pura equivale al tiempo } \\
\text { restante de vida }(\mathrm{t}) \text { : variable incierta }\end{array}$ \\
\hline Plazo del contrato = 10 años & $\begin{array}{l}\text { Precio de ejercicio }(\mathrm{X}) \text { de opción tradicional. En este } \\
\text { caso vencimiento o vigencia del contrato }(\mathrm{T})=10 \text { años }\end{array}$ \\
\hline & $\begin{array}{l}\text { Probabilidad de ejercicio de la opción } \mathrm{N}(-\mathrm{d} 2) \text {. En este } \\
\text { caso, probabilidad condiciónal } \mathrm{t}<\mathrm{T}(\text { tiempo restante } \\
\text { de vida menor al tiempo de vencimiento del contrato) }\end{array}$ \\
Probabilidad de muerte del asegurado & $\begin{array}{l}\text { Esta estimación condenza la información de las dos } \\
\text { variables precedentes }\end{array}$ \\
\hline Monto asegurado fijo $=$ US\$ 10.000 & $\begin{array}{l}\text { Valor intrínseco de la opción cuando esta se encuentra } \\
\text { dentro del dinero. Pago fijo a recibir }(\mathrm{Q})=\mathrm{US \$} 10.000\end{array}$ \\
\hline Tasa garantizada & Tasa libre de riesgo $(\mathrm{Rf})=2 \%$ anual \\
\hline
\end{tabular}

Teniendo en cuenta la definición de parámetros precedente, se presenta un conjunto de casos de valoración del seguro bajo estudio en las subsecciones siguientes, comenzando con una situación 
simplificada y luego presentando extensiones que cambian algunos supuestos que generan resultados más reales y complejos.

\subsection{Caso simple: distribución uniforme continua.}

En este primer caso sencillo, el beneficiario del asegurado cobra el monto fijo en caso de que ocurra el siniestro. Para comenzar con un caso ilustrativo, se propone que el tiempo restante de vida se distribuye continua y uniformemente, en un intervalo definido entre 0 y 75 años, esto es, por encima de la edad actual del asegurado, que claramente alcanza el momento presente con vida (Considerando que el asegurado debe ser mayor de 14 años para contratar el seguro, se estaría definiendo la edad de muerte entre 15 y 90 años (15+75). Si bien la edad del límite superior supera la edad para contratar el seguro de vida de acuerdo a las condiciones de la póliza, la valoración se realiza teniendo en cuenta la probabilidad prorrateada en ese intervalo de tiempo). Esto sería análogo a suponer que la prima del seguro se cotiza nivelada, esto es promediando todos los rangos de edad venideros.

Figura 5. Definición de la variable estocástica bajo una distribución uniforme.

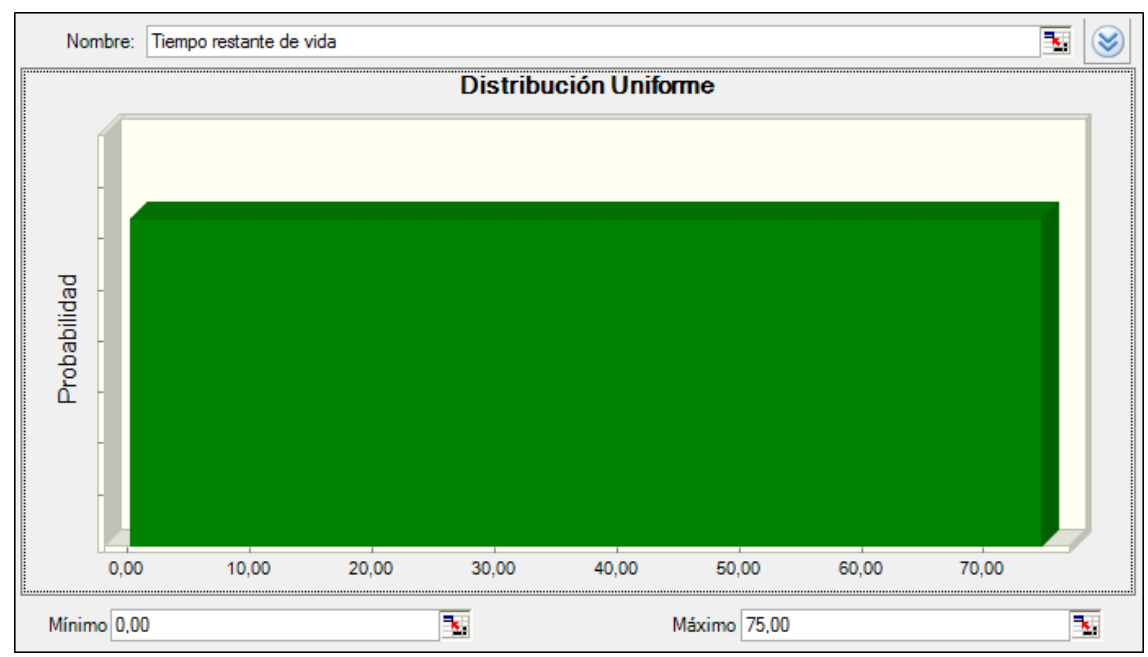

Fuente: Elaboración propia utilizando software de simulación.

Se realizan simulaciones con 1.000.000 de iteraciones para determinar la probabilidad de morir durante el plazo de contrato. Esto es, si la variable incierta "tiempo restante de vida" supera los 10 años, el asegurado no ejerce su derecho por lo que la muerte no ocurre durante la vida del contrato, mientras que la probabilidad del siniestro se computa en caso contrario. La función que determina los casos que mueren se define formalmente como una condicional, donde si $(t>T ; 0 ; 1)$, donde $t$ el tiempo restante de vida, $T$ el vencimiento del contrato y la variable binaria toma valor unitario cuando ocurre el siniestro.

Por ello, a partir de los casos que fallecen durante la vida del contrato mediante la simulación, se calcula un promedio ponderado, alcanzando una probabilidad de muerte acumulada en 10 años de 13,3256\%, como se muestra en la Figura 6. Dicha probabilidad representa las oportunidades de que la opción put digital pura se encuentre dentro del dinero, es decir N(-d2). 
Figura 6. Estimación de la probabilidad de ejercicio con distribución uniforme.

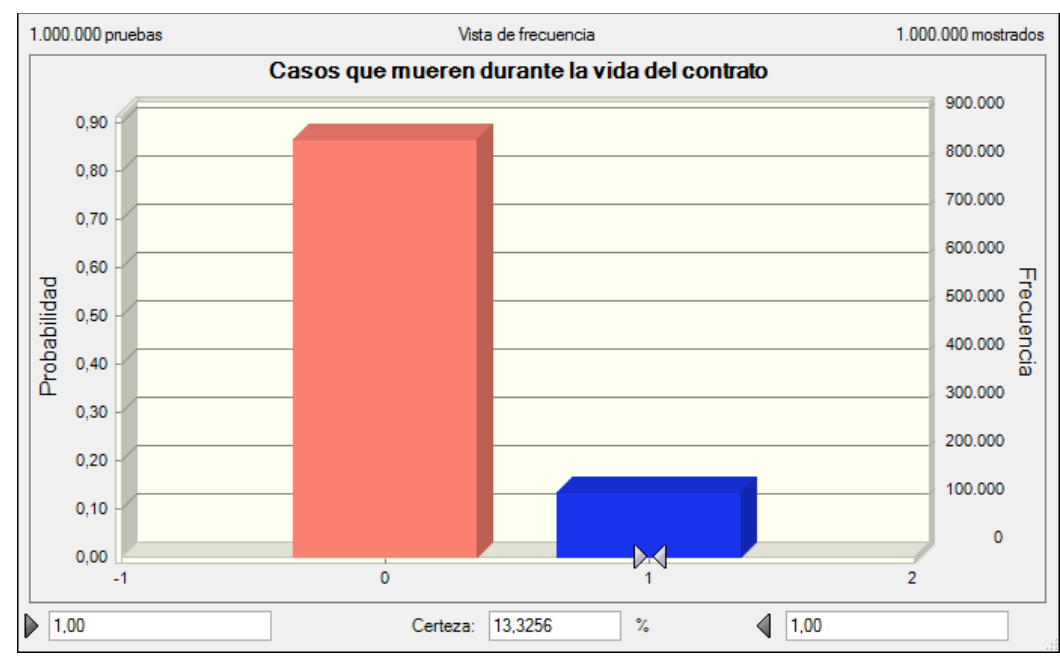

Fuente: Elaboración propia utilizando software de simulación.

Esto permite estimar un valor para la opción digital pura de US\$ 1.091 (Tabla 3), que se trataría de una prima alta para la población relativamente joven, debido a la sobreestimación de la probabilidad de muerte que asigna la distribución uniforme en la etapa activa de la vida; y una prima baja para la población más longeva. Debemos aclarar que éste es el valor del contrato para 10 años, lo que arroja una anualidad equivalente a US\$121,46, o bien US\$10,23 mensuales.

Tabla 3. Inputs y valor según modelo de Black \& Scholes con distribución uniforme.

\begin{tabular}{|c|c|}
\hline $\mathrm{N}(-\mathrm{d} 2)$ & 0,13326 \\
\hline $\mathrm{Q}$ & $\$ 10.000$ \\
\hline $\mathrm{Rf}$ & $2 \%$ \\
\hline $\mathrm{T}$ & 10 \\
\hline Valor opción exótica & US\$ 1091 \\
\hline
\end{tabular}

\subsection{Distribución ajustada a datos actuales de Argentina.}

Teniendo en cuenta la simplificación excesiva que provoca asumir que el tiempo restante de vida se distribuye continua y uniformemente, se presenta a continuación un nuevo caso en donde la distribución estocástica de la variable incierta se ajusta a los datos reales que provee el Ministerio de Salud y Desarrollo Social de la Nación Argentina, en el anuario de 2017 (disponible en: http://www.deis.msal.gov.ar/index.php/anuario-2017/. Fecha de consulta: 31/07/2019). A partir de los datos que se proveen sobre cantidad de muertos por edad en todo el país como jurisdicción, se realizaron los cálculos de las frecuencias para los rangos etarios de interés, es decir, los mayores de 15 años. La ventaja de trabajar con este enfoque es poder prescindir de las tablas de mortalidad provistas por las Superintendencia de Seguros de la Nación y ajustar la distribución a datos locales y actuales de la región bajo estudio. A partir del mencionado análisis se arriba a la distribución de frecuencias que presenta el Gráfico 2. 
Gráfico 2. Frecuencia de mortalidad por rango etario.

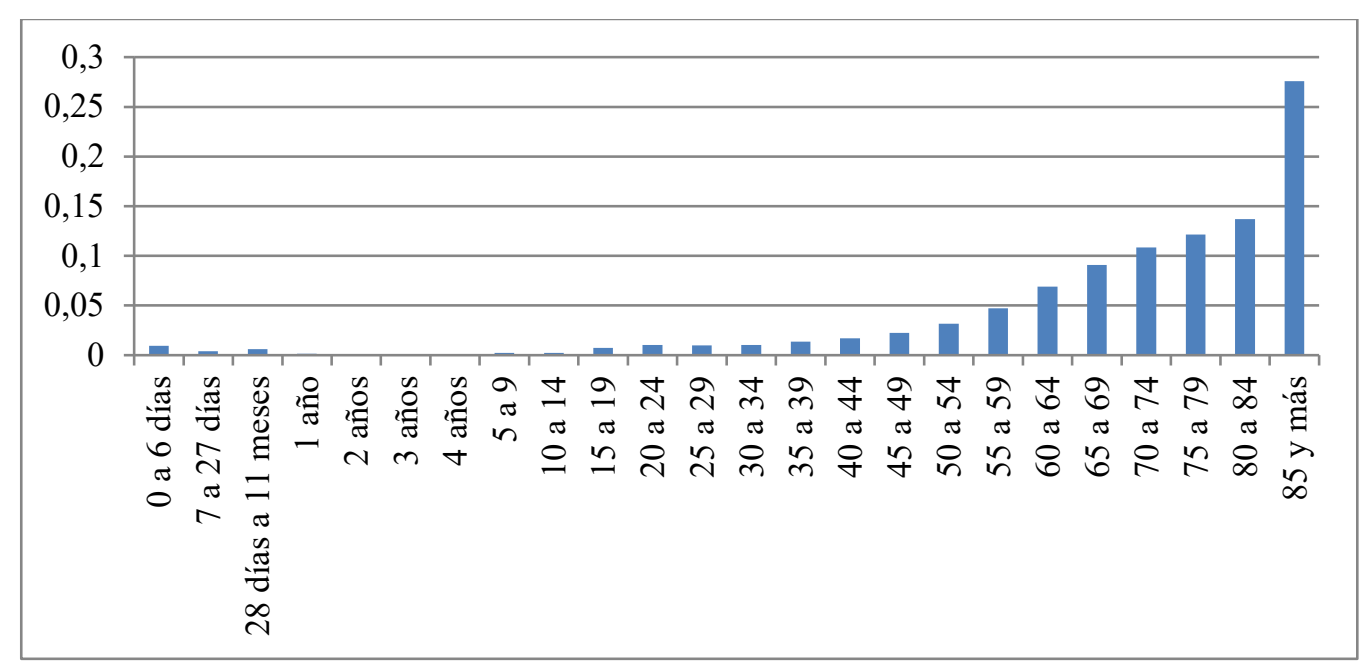

Fuente: Elaboración propia con base en datos de Ministerio de Salud y Desarrollo Social de la Argentina.

A partir de la distribución de frecuencias presentada se define estocásticamente el tiempo de vida restante para un individuo de 15 años (que luego es sensibilizado para edades superiores), mediante una distribución personalizada, continua y creciente hacia la derecha, espejo con la distribución exponencial (Figura 7).

Figura 7. Definición de la variable estocástica bajo una distribución ajustada a los datos.

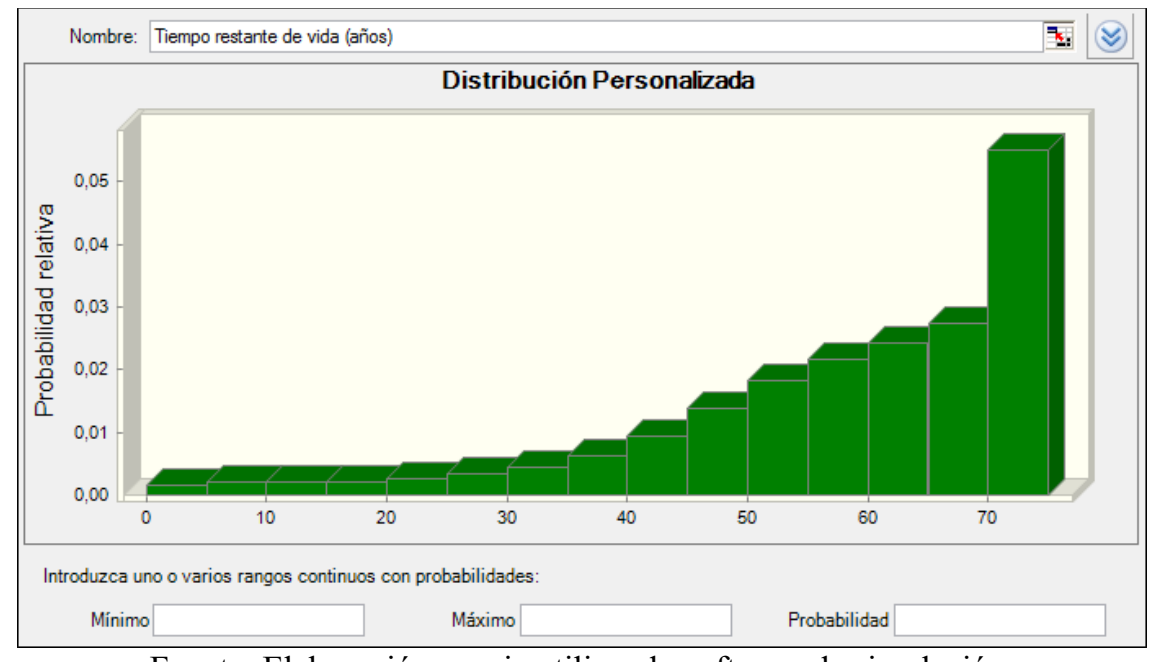

Fuente: Elaboración propia utilizando software de simulación.

Se repite el procedimiento de contabilizar del millón de iteraciones, en cuántos casos se ejercería la opción, es decir, fallecerían dentro del plazo del contrato (10 años). Se recuerda que la función que determina los casos que mueren se define formalmente como una condicional, donde si $(t>T ; 0 ; 1)$. Los resultados se presentan en la Figura 8. 
Figura 8. Estimación de la probabilidad de ejercicio con distribución personalizada.

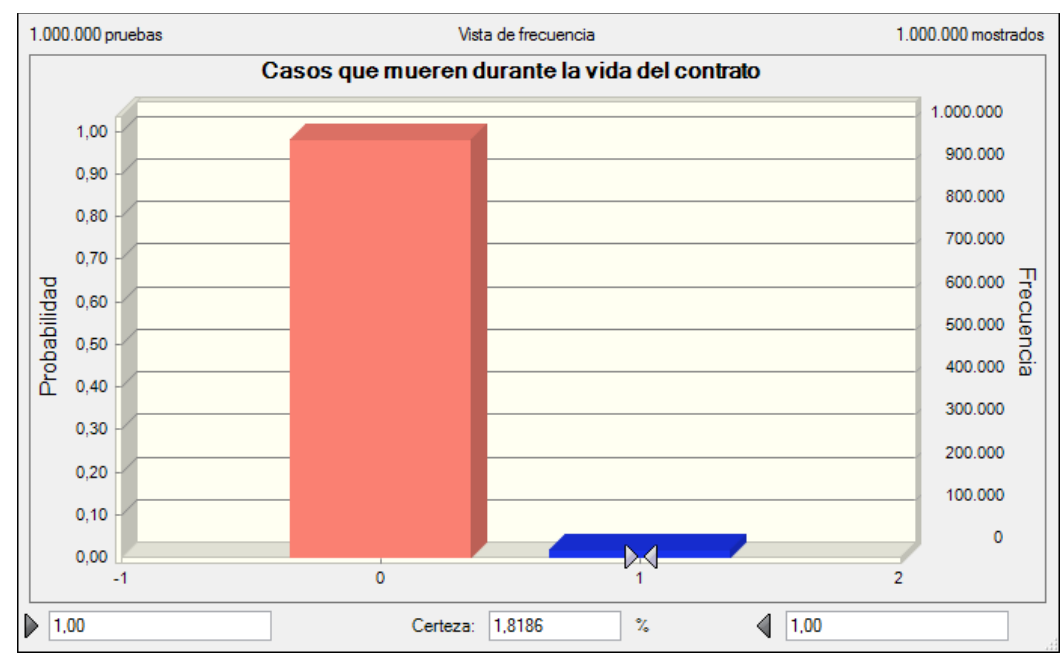

Fuente: Elaboración propia utilizando software de simulación.

La baja probabilidad de ejercicio de la opción put digital pura, de $1,8186 \%$ se debe a la edad de partida del asegurado, un joven que atraviesa la década con menor probabilidad de muerte durante la vida del contrato. A partir de esta probabilidad, que representa $\mathrm{N}(-\mathrm{d} 2)$, se calcula en la siguiente tabla el valor de la opción exótica, que arroja un monto mínimo de US\$149 para los 10 años.

Tabla 4. Inputs y valor según modelo de Black \& Scholes con distribución personalizada.

\begin{tabular}{|c|c|}
\hline $\mathrm{N}(-\mathrm{d} 2)$ & 0,018186 \\
\hline $\mathrm{Q}$ & $\$ 10.000$ \\
\hline $\mathrm{Rf}$ & $2 \%$ \\
\hline $\mathrm{T}$ & 10 \\
\hline Valor opción exótica & US\$ 149 \\
\hline
\end{tabular}

\subsection{Distribución ajustada a datos de Argentina y análisis de sensibilidad según la edad.}

En esta subsección se repite el procedimiento anterior, pero tomando casos en los que el asegurado tiene 30, 40, 50, 60 ó 70 años al momento de la contratación del seguro (este último caso no suele tener disponible la opción de un seguro de vida de acuerdo a las cláusulas de las pólizas analizadas, salvo pacto contrario). Las distribuciones definidas para la variable tiempo restante de vida son ajustadas en cada caso, considerando que llegaron a cada una de esas edades con vida. Los gráficos de suposiciones sobre la variable estocástica y las figuras con la probabilidad de ejercicio con distribución personalizada para cada caso pueden encontrarse en el apéndice de este trabajo (Figuras A.1 a A.10).

Los resultados se resumen en la Tabla 5, donde puede observarse que la probabilidad N(-d2) es creciente cuando se incrementa la edad del asegurado, lo que aumenta la probabilidad del ejercicio de la opción y, por lo tanto, su valor. Sin embargo y como es esperable, las relaciones entre las variables no son lineales sino más bien crecientes exponencialmente (Gráfico 3). 
Tabla 5. Estimación del valor de la opción para diferentes edades del asegurado con distribución personalizada.

\begin{tabular}{ccc}
\hline Si el asegurado tiene & N(-d2) & Valor opción exótica \\
\hline 15 años & $1,8186 \%$ & US\$ 149 \\
\hline 30 años & $2,5338 \%$ & US\$ 207 \\
\hline 40 años & $4,2591 \%$ & US\$ 349 \\
\hline 50 años & $8,9681 \%$ & US\$ 734 \\
\hline 60 años & $19,9163 \%$ & US\$ 1.631 \\
\hline 70 años & $35,7986 \%$ & US\$ 2.931
\end{tabular}

Fuente: Elaboración propia.

Gráfico 3. Relaciones entre la edad, la probabilidad de ejercicio y el valor de la opción.

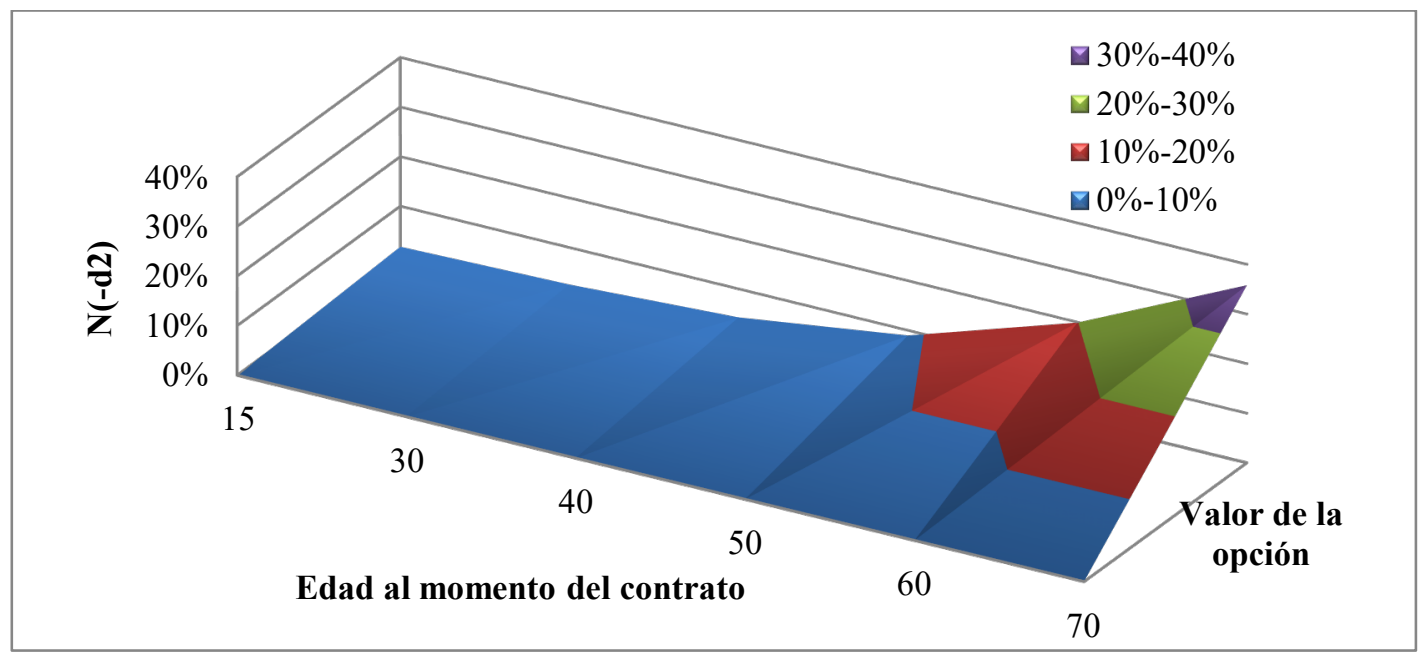

Fuente: Elaboración propia.

Es interesante remarcar que comparando los resultados precedentes con los presentados en el apartado 5.1 en el que se asume una distribución continua uniforme sobre los años de vida residual, la probabilidad de ejercicio y el valor de la opción se encuentran en el rango de valores que se define para los asegurados de entre 50 y 60 años, suponiendo la distribución personalizada que se ajusta a los datos del Ministerio de Salud y Desarrollo Social.

\subsection{Distribución ajustada a datos de Argentina, teniendo en cuenta edad y género.}

Considerando no solo la edad, sino también el género del asegurado, se desagrega el análisis realizado anteriormente. En este caso, con fines ilustrativos se presentan los resultados de un asegurado de 40 años de edad.

También en esta estimación, la distribución estocástica de la variable incierta se ajusta a los datos reales que provee el Ministerio de Salud y Desarrollo Social de la Nación Argentina. A partir de esa información se estima la probabilidad y probabilidad acumulada de fallecimiento por género, según se expone en los Gráficos 4 y 5. 
Gráfico 4. Probabilidad de fallecimiento por género.

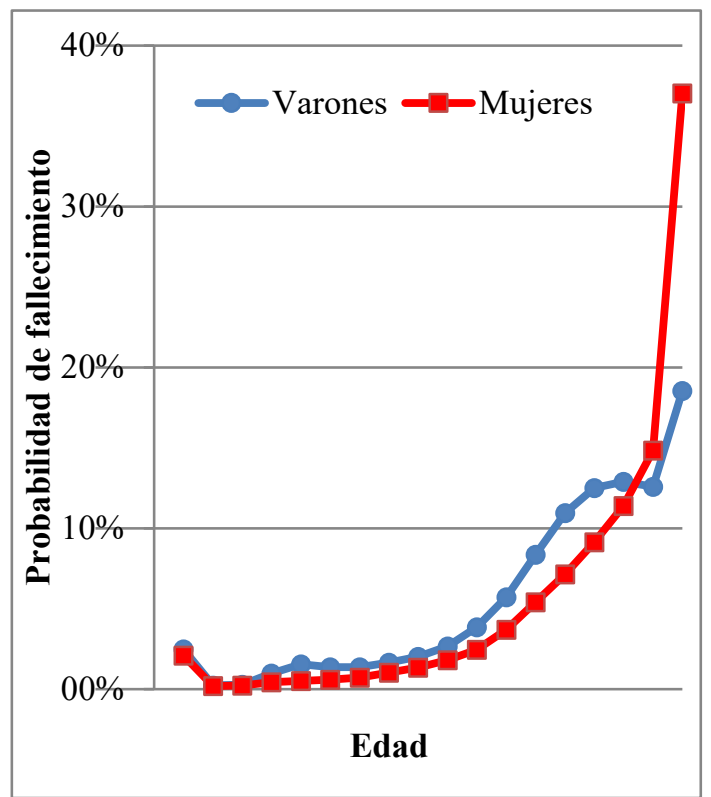

Fuente: Elaboración propia partiendo de datos del Ministerio de Salud y Desarrollo Social de Argentina.
Gráfico 5. Probabilidad acumulada de fallecimiento por género.

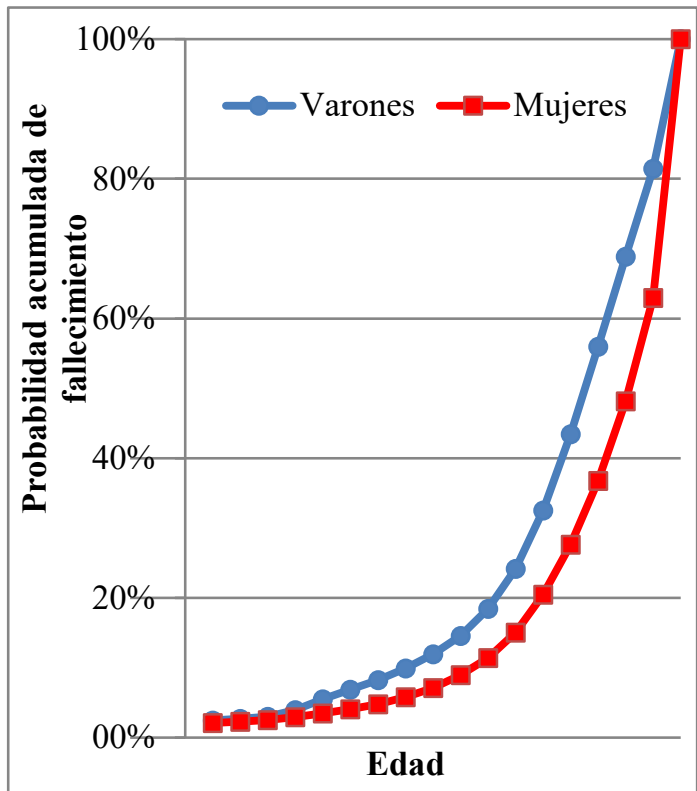

Fuente: Elaboración propia partiendo de datos del Ministerio de Salud y Desarrollo Social de Argentina.

Como puede observarse, el patrón de mortalidad de los hombres presenta un incremento de la probabilidad de fallecimiento a edades más tempranas, cuestión que se compensa para adultos mayores.

A partir de los datos, se realizan las estimaciones para definir las distribuciones de probabilidad personalizadas por género, según se expone en las figuras del apéndice del trabajo (A.11 a A.14). De este modo, para un asegurado de 40 años de edad puede estimarse una probabilidad de mortalidad durante la vigencia de la póliza de 10 años de $5,17 \%$ en caso de los individuos de género masculino, mientras que la misma es de 3,33\% para el género femenino. Esto arroja valores para la opción de US\$ 423 y US\$ 273 respectivamente (Tabla 6), lo que representa una variación de entre 21 y $22 \%$ respecto al caso base en el que no se diferenciaba el género del asegurado.

Tabla 6. Estimación del valor de la opción para asegurado según género con distribución personalizada.

\begin{tabular}{lccc}
\hline \multicolumn{1}{c}{ Si el asegurado es } & N(-d2) & Valor opción exótica & $\Delta$ caso base \\
\hline Masculino con 40 años & $5,1701 \%$ & US\$ 423 & $+21,2 \%$ \\
\hline Femenino con 40 años & $3,3305 \%$ & US\$ 273 & $-21,7 \%$ \\
\hline Caso general 40 años & $4,2591 \%$ & US\$ 349 & $0,0 \%$ \\
\hline
\end{tabular}

Fuente: Elaboración propia

\subsection{Comparación de los resultados con las primas de mercado.}

Los valores encontrados en este análisis de casos mediante opciones exóticas son ajustados y comparados con pólizas de seguro contratadas en el ámbito local. Para tal fin se procede con un cálculo intermedio de proporcionar la prima estimada para 10 años a un horizonte temporal de un año, a partir 
de asumir que el valor teórico corresponde al monto actualizado de la prima anual, descontando los flujos a la tasa libre de riesgo en dólares presentada en las secciones anteriores. Al valor de la prima anual se le agrega un monto de $13,31 \%$ en concepto de cargos financieros y administrativos, que es el porcentaje promedio que representan estos conceptos sobre la prima, con base en siete contratos de seguros de vida analizados (dicho porcentaje ronda específicamente entre 11,64\% y 14,22\%).

Tabla 7. Ajustes sobre el valor teórico estimado a los fines de su comparación.

\begin{tabular}{crr}
\hline Si el asegurado es & Concepto & \multicolumn{1}{c}{ Monto } \\
\hline \multirow{3}{*}{ Masculino con 40 años } & Valor prima 10 años & US\$ 423,00 \\
& Valor prima anual & US\$ 47,09 \\
& Valor premio anual & US\$ 53,36 \\
\hline \multirow{2}{*}{ Femenino con 40 años } & Valor prima 10 años & US\$ 273,00 \\
& Valor prima anual & US\$ 30,39 \\
& Valor premio anual & US\$ 34,44 \\
\hline
\end{tabular}

Fuente: Elaboración propia.

Debido a la confidencialidad de los datos, las pólizas no son presentadas individualmente en este trabajo, pero corresponden a siete contratos de cuatro compañías aseguradoras locales privadas. De manera agregada y a través de promedios simples para las condiciones del sujeto informadas y un monto asegurado equivalente a US\$10.000, en la Tabla 8 se presentan los resultados comparativos para dos sujetos de edad media.

Tabla 8. Comparación de valores teóricos y premio de mercado.

\begin{tabular}{lcc}
\hline Condición del sujeto & Estimación teórica & Premio de mercado \\
\hline Masculino con 40 años & US\$ 53,36 & US\$ 114,75 \\
\hline Femenino con 40 años & US\$ 34,44 & US\$ 106,50 \\
\hline
\end{tabular}

Fuente: Elaboración propia.

En términos generales, los valores de mercado superan en más del doble a los valores teóricos estimados. Cabe recordar que, teniendo en cuenta que los casos desarrollados en las secciones previas no consideran cargos financieros ni administrativos, los valores de las primas puras fueron ajustados para incoporarlos (Tabla 7). A efectos de mejorar la comparabilidad, se ajustó la prima por el monto asegurado y se consideró el rango etario al que pertenecía el individuo en particular.

Los resultados obtenidos entre las primas teóricas y de mercado arrojan diferencias por diversos motivos potenciales que se resumen en la Tabla 9, entre los que se pueden destacar que dentro del valor teórico alcanzado, no se encuentra incluida ningún tipo de rentabilidad para la empresa aseguradora, cuestión que sí incorporan las pólizas de mercado. Esta discrepancia suavizaría la diferencia entre el valor teórico y de mercado, pero dada la magnitud del diferencial encontrado, difícilmente se revierte la relación entre los valores. Entre otros posibles motivos sobre los factores que pueden llevar a esta sobrevaloración en las primas observadas se resumen: las diferencias en las estimaciones de las probabilidades de muerte, el tamaño de la muestra de pólizas analizadas y su circunscripción a un área local, el incentivo generado por la rentabilidad de los fondos acumulados del asegurado y el potencial retiro de ellos (opción de salida). En futuros trabajos de investigación se pretende abordar el análisis del valor añadido producto de la opcionalidad contenida en este tipo de contratos. 
Tabla 9. Causas potenciales de las diferencias entre valor teórico y de mercado.

\begin{tabular}{|c|c|}
\hline Origen de la causa & Observaciones \\
\hline \multirow[t]{2}{*}{$\begin{array}{l}\text { Características de los } \\
\text { datos utilizados }\end{array}$} & $\begin{array}{l}\text { Con la intención de realizar una estimación con fuentes alternativas, } \\
\text { y tal como se relató previamente, no se utilizaron las tablas } \\
\text { actuariales suministradas por la Superintendencia de Seguros de la } \\
\text { Nación. Esto puede provocar divergencias dado que los datos } \\
\text { probabilísticos utilizados se calculan a partir de cifras oficiales, } \\
\text { informadas por el Ministerio de Salud y Desarrollo Social de la } \\
\text { Nación Argentina, y si bien son datos de la zona geográfica del } \\
\text { estudio, podrían existir diferencias en la probabilidad de muerte } \\
\text { respecto a la población que contrata un seguro de este tipo, por la } \\
\text { condición socio-económica y biométrica de origen, aunque esto } \\
\text { reduciría aún más la prima teórica estimada, incrementando la } \\
\text { diferencia de la Tabla } 8 \text {. }\end{array}$ \\
\hline & $\begin{array}{l}\text { Por otro lado, que los valores de mercado se basen en una muestra } \\
\text { no probabilística de siete contratos provenientes de cuatro compañías } \\
\text { aseguradoras locales, no asegura que los valores sean generalizables } \\
\text { a la población de contratos de seguros de vida suscriptos en el país. }\end{array}$ \\
\hline Método de valoración & $\begin{array}{l}\text { El uso de modelos de valoración de opciones financieras para el } \\
\text { cálculo de la prima teórica, en lugar de la utilización del cálculo } \\
\text { actuarial tradicional puede ser causa de divergencias. Este último es } \\
\text { objetivo en probabilidades que se ajustan al perfil del tomador, pero } \\
\text { no se incorpora flexibilidad estratégica, dado que su cálculo consiste } \\
\text { en un valor actual simple. }\end{array}$ \\
\hline $\begin{array}{l}\text { Margen de rentabilidad } \\
\text { de } \\
\text { aseguradoras }\end{array}$ & $\begin{array}{l}\text { La rentabilidad de la empresa aseguradora no es considerada en el } \\
\text { análisis previo. La incorporación de este concepto incrementaría el } \\
\text { monto teórico, reduciendo la diferencia entre los valores presentados } \\
\text { en la Tabla } 8 .\end{array}$ \\
\hline $\begin{array}{l}\text { Otras opciones que } \\
\text { presenta el contrato de } \\
\text { seguro }\end{array}$ & $\begin{array}{l}\text { Los movimientos del fondo acumulado y la opción de salida del } \\
\text { seguro de vida no se incorporaron en el análisis con opciones } \\
\text { exóticas. } \\
\text { El hecho de que la prima se convierta en una inversión financiera } \\
\text { podría provocar un incremento de magnitud, debido a la rentabilidad } \\
\text { esperada de fondo, moderando la diferencia entre los valores teóricos } \\
\text { y de mercado de la Tabla } 8 \text {. }\end{array}$ \\
\hline
\end{tabular}

Fuente: Elaboración propia.

\section{Conclusiones.}

El trabajo realiza una valoración teórica de un seguro de vida analizándolo como una opción exótica del estilo digital pura o pulso, también denominada cash or nothing. La misma se evalúa a través del método del Black-Scholes ajustado, para diferentes escenarios de datos demográficos de los asegurados y realizando simulaciones de Monte Carlo para modelar la distribución de probabilidad de la vida residual del individuo al momento de la suscripción de la póliza. Los resultados permiten complementar el análisis actuarial, iluminando los efectos explícitos que provocan los cambios en las condiciones del asegurado y su ajuste de la función de probabilidad de sobrevida. Como es esperable, para individuos de menor edad y género femenino se obtienen los contratos con menores costos. El valor de la opción aumenta al sensibilizar la edad para asegurados mayores, creciendo exponencialmente debido al incremento de la probabilidad de ejercicio $(\mathrm{N}(-\mathrm{d} 2))$. 
Dentro de las limitaciones del trabajo se puede mencionar que, si bien el modelo de BlackScholes-Merton es el más utilizado para valorar opciones financieras, podría suceder que los supuestos propios del método sean demasiado contundentes para aplicarlos a opciones exóticas de este tipo, aunque el modelo fue adaptado en su aplicación. Asimismo, se testearon algunas distribuciones de probabilidad para la variable estocástica, estando pendiente el análisis de distribuciones alternativas y el uso modelos más explícitos y adaptables como el binomial.

Como futura línea de investigación es de interés analizar el valor de los seguros de vida con opcionalidad, por ejemplo, con posibilidad de retiro del fondo acumulado. En estos casos, aparecería una nueva opción exótica, en simultáneo con la digital pura, del estilo asiática, en las que a partir de un determinado momento de tiempo se puede retirar el fondo acumulado o dejarlo invertido en la aseguradora para que siga capitalizando. La regla de decisión del asegurado es retirar el fondo cuando el valor actual del mismo sea superior al valor esperado del pago fijo de la opción digital, capitalizado a la misma tasa.

Asimismo, se pretende ampliar el análisis de seguros de vida en Argentina y realizar la comparación con otros países de la región, en los que este tipo de contratos presenten mayor relevancia en la industria aseguradora.

\section{Agradecimientos}

Este artículo fue desarrollado en el marco del Proyecto de Grupo de Investigación sobre "Derivados exóticos aplicados a problemas de valuación de activos no financieros", dirigido por la Dra. Gabriela Pesce con la co-dirección del Dr. Gastón Milanesi (PGI 24/C054).

\section{Referencias}

Black, F., \& Scholes, M. (1973). The pricing of options and corporate liabilities. Journal of Political Economy, 81(3), 637-654. https://doi.org/10.1086/260062

Boletín Oficial de la República Argentina (2018). Legislación y Avisos Oficiales. Superintendencia de Seguros de la Nación. Resolución 697/2018. Recuperado de https://www.boletinoficial.gob.ar/detalleAviso/primera/188883/20180726.

Breslow, N., \& Crowley, J. (1974). A Large Sample Study of the Life Table and Product Limit Estimates under Random Censorship. The Annals of Statistics, 2(3), 437-453. https://doi.org/10.1214/aos/1176342705

Casparri, M.T., \& García, J. (2010). Algunas innovaciones financieras para La gestión del riesgo global. Buenos Aires: Editorial de la Facultad de Ciencias Económicas de la Universidad de Buenos Aires, Argentina.

Cummins, J.D. (1990). Asset pricing models and insurance ratemaking. ASTIN Bulletin: The Journal of the IAA, 20(2), 125-166. https://doi.org/10.2143/AST.20.2.2005438

Cummins, J.D. (1991). Statistical and financial models of insurance pricing and the insurance firm. The Journal of Risk and Insurance, 58(2), 261-302. https://doi.org/10.2307/253237

D'Arcy, S.P., \& Doherty, N.A. (1988). The financial theory of pricing property-liability insurance contracts. SS Huebner Foundation for Insurance Education, Wharton School, University of Pennsylvania. https://doi.org/10.2143/ast.18.2.2014953 
del Pozo, E.M. (2001). Modelos de opciones aplicados al seguro (No. 01-09). Madrid: Universidad Complutense de Madrid, Facultad de Ciencias Económicas y Empresariales.

Fernández, P., \& Ariño, M.A. (1996). Derivados exóticos. Documento de investigación 308. Centro Internacional de Investigación Financiera (CIIF) y División de Investigación IESE, Universidad de Navarra.

Gaarder, E. (2007). Derivatives: Models on Models. Chichester, United Kingdom: John Wiley \& Sons.

García, J.J., Sancha, M.P., Tejero, C., \& Toscano, D. (2000). Opciones “exóticas". Boletín Económico de ICE, 2673, 1-8.

Gatzert, N., \& Schmeiser, H. (2006). Implicit options in life insurance: Valuation and risk management. Zeitschrift für die gesamte Versicherungswissenschaft,95(1), 111-128. https://doi.org/10.1007/BF03353443

Gompertz, B. (1825). On the Nature of the Function Expressive of the Law of Human Mortality, and on a New Mode of Determining the Value of Life Contingencies. Philosophical Transactions of the Royal Society of London, 115, 513-583. https://doi.org/10.1098/rstl.1825.0026

Hull, J. (2012). Options, Futures and other Derivatives (12 ${ }^{\mathrm{a}}$ ed.). Harlow, United Kingdom: Pearson Education Global Edition.

Hull, J. (2014). Introducción a los mercados de futuros y opciones ( $8^{\mathrm{a}}$ ed.). Madrid, España: Editorial Pearson.

Kolb, R. (2003). Futures, Options, and Swaps. Oxford, United Kingdom: Blackwell Publishing.

Kolb, R., \& Overdahl, J. (2010). Financial derivatives. Pricing and risk management. New Jersey, United States: John Wiley \& Sons.

Ley No 17.418 (1967). Ley de Seguros. Boletín Oficial de la República, Buenos Aires, Argentina, 30 de agosto de 1967.

Luoma, A., Puustelli, A., \& Koskinen, L. (2008). Bayesian analysis of participating life insurance contracts with American-style options. In Proceedings of AFIR Colloquium. http://www.actuaries.org/AFIR/Colloquia/Rome2/Koskinen_Luoma_Puustelli.pdf

Makeham, W.M. (1860). On the Law of Mortality and the Construction of Annuity Tables. The Assurance Magazine and Journal of the Institute of Actuaries, 8(6), 301-310. https://doi.org/10.1017/s204616580000126x

Melinsky, Pellegrinelli y Asoc. (2019). Tablas de mortalidad. Recuperado de http://www.melpel.com.ar/?p=678. Fecha de consulta: 01/08/2019.

Merton, R.C. (1973). Theory of rational option pricing. Bell Journal of Economics and Management Science, 4(1), 141-183. https://doi.org/10.2307/3003143

National Vital Statistics Report (2006). United States Life Tables 2003, by Elizabeth Arias. National Vital Statistics Reports, 54(14), 1-40. https://www.cdc.gov/nchs/data/nvsr/nvsr54/nvsr54_14.pdf

Whaley, R. (2006). Derivatives: Markets, Valuation and Risk Management. New Jersey, United States: John Wiley \& Sons. 
Wilmott, P. (2009). Frequently Asked Questions in Quantitative Finance. London, United Kingdom: John Wiley \& Sons.

Yin, R. (2005). Estudo de caso: planejamento e métodos ( $3^{\mathrm{a}}$ ed.). Porto Alegre, Brasil: Bookman. 


\section{Apéndices}

Tabla A.1. Mortalidad según Commisioner Standard Ordinary 2001.

\begin{tabular}{|c|c|c|c|c|c|c|}
\hline \multirow[b]{3}{*}{ Edad } & \multicolumn{6}{|c|}{ Probabilidad de fallecimiento - $\mathbf{q}(\mathbf{x}) * 1000$} \\
\hline & & Hombres & & & Mujeres & \\
\hline & General & No Fumador & Fumador & General & No Fumador & Fumador \\
\hline 0 & 0.970 & & & 0.480 & & \\
\hline 1 & 0.560 & & & 0.350 & & \\
\hline 2 & 0.390 & & & 0.260 & & \\
\hline 3 & 0.270 & & & 0.200 & & \\
\hline 4 & 0.210 & & & 0.190 & & \\
\hline 5 & 0.210 & & & 0.180 & & \\
\hline 6 & 0.220 & & & 0.180 & & \\
\hline 7 & 0.220 & & & 0.210 & & \\
\hline 8 & 0.220 & & & 0.210 & & \\
\hline 9 & 0.230 & & & 0.210 & & \\
\hline 10 & 0.230 & & & 0.220 & & \\
\hline 11 & 0.270 & & & 0.230 & & \\
\hline 12 & 0.330 & & & 0.270 & & \\
\hline 13 & 0.390 & & & 0.300 & & \\
\hline 14 & 0.470 & & & 0.330 & & \\
\hline 15 & 0.610 & & & 0.350 & & \\
\hline 16 & 0.740 & 0.740 & 0.790 & 0.390 & 0.390 & 0.410 \\
\hline 17 & 0.870 & 0.850 & 0.970 & 0.410 & 0.410 & 0.460 \\
\hline 18 & 0.940 & 0.920 & 1.110 & 0.430 & 0.420 & 0.500 \\
\hline 19 & 0.980 & 0.940 & 1.210 & 0.460 & 0.450 & 0.540 \\
\hline 20 & 1.000 & 0.950 & 1.270 & 0.470 & 0.450 & 0.580 \\
\hline 21 & 1.000 & 0.950 & 1.330 & 0.480 & 0.460 & 0.610 \\
\hline 22 & 1.020 & 0.950 & 1.400 & 0.500 & 0.480 & 0.650 \\
\hline 23 & 1.030 & 0.960 & 1.460 & 0.500 & 0.480 & 0.670 \\
\hline 24 & 1.050 & 0.970 & 1.540 & 0.520 & 0.500 & 0.720 \\
\hline 25 & 1.070 & 0.980 & 1.630 & 0.540 & 0.500 & 0.770 \\
\hline 26 & 1.120 & 1.020 & 1.710 & 0.560 & 0.530 & 0.810 \\
\hline 27 & 1.170 & 1.070 & 1.810 & 0.600 & 0.570 & 0.870 \\
\hline 28 & 1.170 & 1.050 & 1.820 & 0.630 & 0.580 & 0.920 \\
\hline 29 & 1.150 & 1.030 & 1.810 & 0.660 & 0.620 & 0.990 \\
\hline 30 & 1.140 & 1.020 & 1.800 & 0.680 & 0.640 & 1.030 \\
\hline 31 & 1.130 & 1.010 & 1.800 & 0.730 & 0.680 & 1.120 \\
\hline 32 & 1.130 & 1.010 & 1.820 & 0.770 & 0.720 & 1.190 \\
\hline 33 & 1.150 & 1.040 & 1.870 & 0.820 & 0.760 & 1.280 \\
\hline 34 & 1.180 & 1.060 & 1.940 & 0.880 & 0.820 & 1.390 \\
\hline 35 & 1.210 & 1.090 & 2.000 & 0.970 & 0.890 & 1.530 \\
\hline 36 & 1.280 & 1.150 & 2.110 & 1.030 & 0.950 & 1.650 \\
\hline 37 & 1.340 & 1.200 & 2.230 & 1.110 & 1.030 & 1.790 \\
\hline 38 & 1.440 & 1.290 & 2.400 & 1.170 & 1.070 & 1.880 \\
\hline 39 & 1.540 & 1.370 & 2.570 & 1.230 & 1.130 & 2.000 \\
\hline 40 & 1.650 & 1.460 & 2.770 & 1.300 & 1.200 & 2.120 \\
\hline 41 & 1.790 & 1.580 & 3.030 & 1.380 & 1.270 & 2.260 \\
\hline 42 & 1.960 & 1.730 & 3.330 & 1.480 & 1.350 & 2.430 \\
\hline 43 & 2.150 & 1.900 & 3.690 & 1.590 & 1.450 & 2.630 \\
\hline 44 & 2.390 & 2.100 & 4.120 & 1.720 & 1.570 & 2.860 \\
\hline 45 & 2.650 & 2.330 & 4.570 & 1.870 & 1.710 & 3.130 \\
\hline 46 & 2.900 & 2.550 & 4.990 & 2.050 & 1.870 & 3.430 \\
\hline 47 & 3.170 & 2.790 & 5.460 & 2.270 & 2.070 & 3.810 \\
\hline 48 & 3.330 & 2.930 & 5.720 & 2.500 & 2.290 & 4.280 \\
\hline 49 & 3.520 & 3.090 & 6.020 & 2.780 & 2.530 & 4.810 \\
\hline 50 & 3.760 & 3.320 & 6.450 & 3.080 & 2.810 & 5.390 \\
\hline 51 & 4.060 & 3.590 & 6.960 & 3.410 & 3.120 & 6.020 \\
\hline 52 & 4.470 & 3.960 & 7.660 & 3.790 & 3.470 & 6.710 \\
\hline 53 & 4.930 & 4.360 & 8.450 & 4.200 & 3.850 & 7.440 \\
\hline 54 & 5.500 & 4.870 & 9.440 & 4.630 & 4.250 & 8.240 \\
\hline 55 & 6.170 & 5.500 & 10.560 & 5.100 & 4.680 & 9.080 \\
\hline 56 & 6.880 & 6.140 & 11.700 & 5.630 & 5.180 & 9.980 \\
\hline 57 & 7.640 & 6.830 & 12.910 & 6.190 & 5.700 & 10.940 \\
\hline 58 & 8.270 & 7.420 & 13.860 & 6.800 & 6.260 & 11.870 \\
\hline 59 & 8.990 & 8.100 & 14.960 & 7.390 & 6.820 & 12.900 \\
\hline 60 & 9.860 & 8.920 & 16.290 & 8.010 & 7.400 & 13.970 \\
\hline
\end{tabular}




\begin{tabular}{|c|c|c|c|c|c|c|}
\hline 61 & 10.940 & 9.920 & 17.940 & 8.680 & 8.030 & 15.080 \\
\hline 62 & 12.250 & 11.140 & 19.930 & 9.390 & 8.720 & 16.330 \\
\hline 63 & 13.710 & 12.510 & 22.140 & 10.140 & 9.430 & 17.580 \\
\hline 64 & 15.240 & 13.950 & 24.400 & 10.960 & 10.200 & 18.900 \\
\hline 65 & 16.850 & 15.470 & 26.630 & 11.850 & 11.050 & 20.340 \\
\hline 66 & 18.470 & 17.010 & 28.780 & 12.820 & 11.990 & 21.870 \\
\hline 67 & 20.090 & 18.570 & 30.870 & 13.890 & 13.020 & 23.590 \\
\hline 68 & 21.850 & 20.250 & 33.070 & 15.070 & 14.170 & 25.480 \\
\hline 69 & 23.640 & 21.990 & 35.250 & 16.360 & 15.430 & 27.530 \\
\hline 70 & 25.770 & 24.100 & 37.890 & 17.810 & 16.820 & 29.820 \\
\hline 71 & 28.150 & 26.460 & 40.780 & 19.470 & 18.420 & 32.430 \\
\hline 72 & 31.320 & 29.560 & 44.710 & 21.300 & 20.210 & 35.310 \\
\hline 73 & 34.620 & 32.830 & 48.660 & 23.300 & 22.150 & 38.410 \\
\hline 74 & 38.080 & 36.270 & 52.650 & 25.500 & 24.280 & 41.810 \\
\hline 75 & 41.910 & 40.030 & 57.290 & 27.900 & 26.640 & 45.230 \\
\hline 76 & 46.080 & 44.130 & 62.230 & 30.530 & 29.230 & 48.960 \\
\hline 77 & 50.920 & 48.890 & 67.940 & 33.410 & 32.080 & 52.970 \\
\hline 78 & 56.560 & 54.450 & 74.540 & 36.580 & 35.230 & 57.290 \\
\hline 79 & 63.060 & 60.870 & 82.050 & 40.050 & 38.630 & 61.960 \\
\hline 80 & 70.140 & 67.870 & 90.070 & 43.860 & 42.430 & 66.990 \\
\hline 81 & 78.190 & 75.840 & 99.050 & 49.110 & 47.590 & 74.070 \\
\hline 82 & 86.540 & 84.140 & 108.110 & 54.950 & 53.410 & 81.760 \\
\hline 83 & 95.510 & 93.090 & 117.610 & 60.810 & 59.210 & 89.250 \\
\hline 84 & 105.430 & 103.000 & 127.940 & 67.270 & 65.620 & 97.300 \\
\hline 85 & 116.570 & 114.070 & 140.090 & 74.450 & 72.840 & 105.410 \\
\hline 86 & 128.910 & 126.340 & 153.390 & 80.990 & 79.390 & 112.170 \\
\hline 87 & 142.350 & 139.740 & 167.690 & 90.790 & 89.250 & 122.890 \\
\hline 88 & 156.730 & 154.100 & 182.720 & 101.070 & 99.550 & 133.590 \\
\hline 89 & 171.880 & 169.250 & 198.270 & 112.020 & 110.530 & 144.350 \\
\hline 90 & 187.660 & 185.060 & 214.130 & 121.920 & 120.650 & 153.050 \\
\hline 91 & 202.440 & 199.930 & 228.430 & 126.850 & 125.770 & 154.940 \\
\hline 92 & 217.830 & 215.430 & 243.020 & 136.880 & 135.840 & 162.660 \\
\hline 93 & 234.040 & 231.780 & 258.100 & 151.640 & 150.780 & 175.100 \\
\hline 94 & 251.140 & 249.050 & 273.740 & 170.310 & 169.640 & 190.970 \\
\hline 95 & 269.170 & 267.190 & 291.050 & 193.660 & 192.920 & 214.970 \\
\hline 96 & 285.640 & 283.790 & 306.330 & 215.660 & 215.030 & 236.910 \\
\hline 97 & 303.180 & 301.490 & 322.440 & 238.480 & 237.790 & 258.950 \\
\hline 98 & 321.880 & 320.380 & 339.450 & 242.160 & 241.690 & 260.010 \\
\hline 99 & 341.850 & 340.540 & 357.420 & 255.230 & 254.740 & 270.770 \\
\hline 100 & 363.190 & 362.100 & 376.400 & 275.730 & 275.460 & 289.270 \\
\hline 101 & 380.080 & 379.210 & 390.770 & 297.840 & 297.550 & 309.030 \\
\hline 102 & 398.060 & 397.440 & 405.920 & 322.210 & 322.190 & 330.780 \\
\hline 103 & 417.200 & 416.840 & 421.830 & 349.060 & 349.040 & 354.110 \\
\hline 104 & 437.560 & 437.480 & 438.570 & 378.610 & 378.600 & 379.410 \\
\hline 105 & 459.210 & 459.130 & 460.150 & 410.570 & 410.560 & 411.320 \\
\hline 106 & 482.220 & 482.150 & 483.100 & 443.330 & 443.320 & 444.020 \\
\hline 107 & 506.690 & 506.620 & 507.510 & 476.890 & 476.880 & 477.530 \\
\hline 108 & 532.690 & 532.630 & 533.440 & 510.650 & 510.640 & 511.240 \\
\hline 109 & 560.310 & 560.260 & 561.010 & 545.810 & 545.800 & 546.350 \\
\hline 110 & 589.640 & 589.590 & 590.270 & 581.770 & 581.760 & 582.260 \\
\hline 111 & 620.790 & 620.740 & 621.350 & 616.330 & 616.320 & 616.780 \\
\hline 112 & 653.840 & 653.800 & 654.350 & 649.850 & 649.840 & 650.250 \\
\hline 113 & 688.940 & 688.910 & 689.380 & 680.370 & 680.360 & 680.720 \\
\hline 114 & 726.180 & 726.150 & 726.560 & 723.390 & 723.380 & 723.690 \\
\hline 115 & 765.700 & 765.670 & 766.010 & 763.410 & 763.400 & 763.660 \\
\hline 116 & 807.610 & 807.590 & 807.860 & 804.930 & 804.920 & 805.120 \\
\hline 117 & 852.070 & 852.050 & 852.260 & 850.440 & 850.440 & 850.590 \\
\hline 118 & 899.230 & 899.220 & 899.350 & 892.440 & 892.440 & 892.540 \\
\hline 119 & 949.220 & 949.220 & 949.290 & 935.110 & 935.110 & 935.160 \\
\hline 120 & 1000.000 & 1000.000 & 1000.000 & 1000.000 & 1000.000 & 1000.000 \\
\hline
\end{tabular}

Fuente: Elaborado por Melinsky, Pellegrinelli \& Asoc. (2019). 
Figuras A.1 a A.14: Distribuciones estocásticas y probabilidad de ejercicio.

Distribución de vida residual

A.1. Distribución si edad $=30$

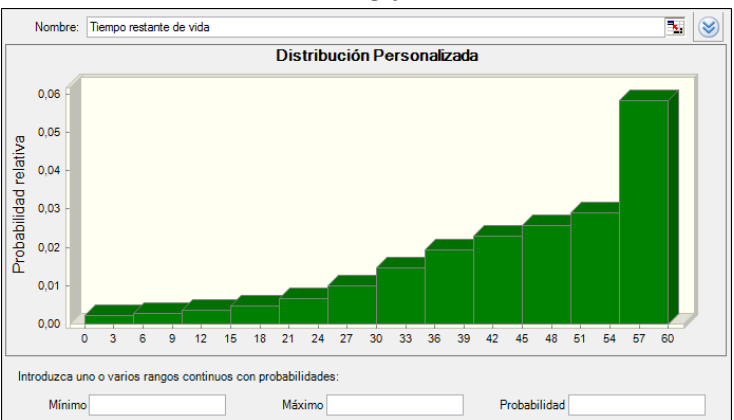

A.3. Distribución si edad $=40$

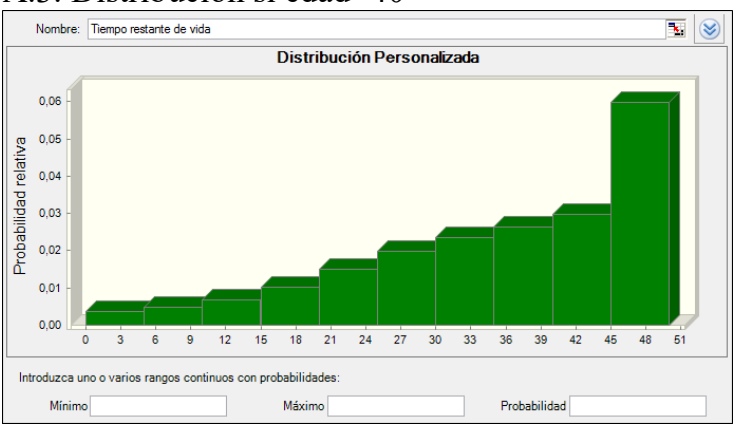

A.5. Distribución si edad $=50$

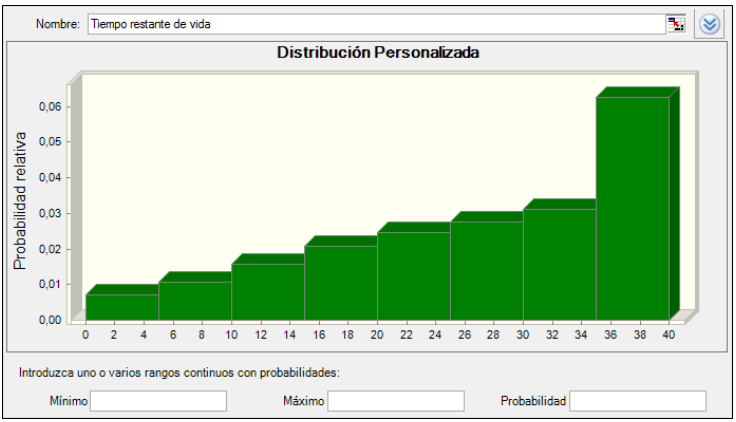

A.7. Distribución si edad $=60$

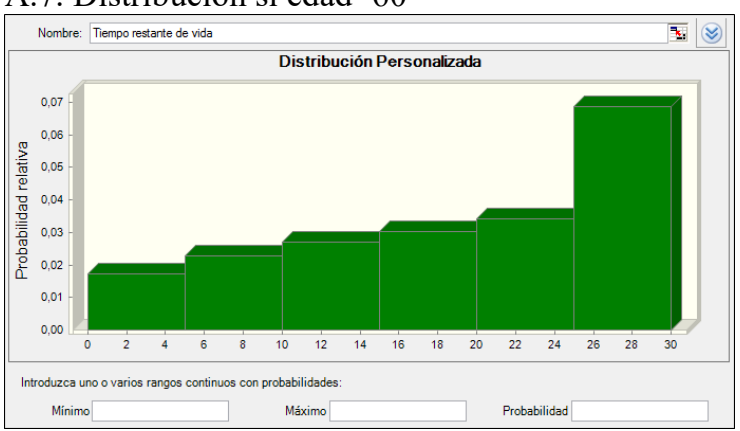

Estimación de la probabilidad de ejercicio

A.2. Probabilidad de ejercicio si edad $=30$

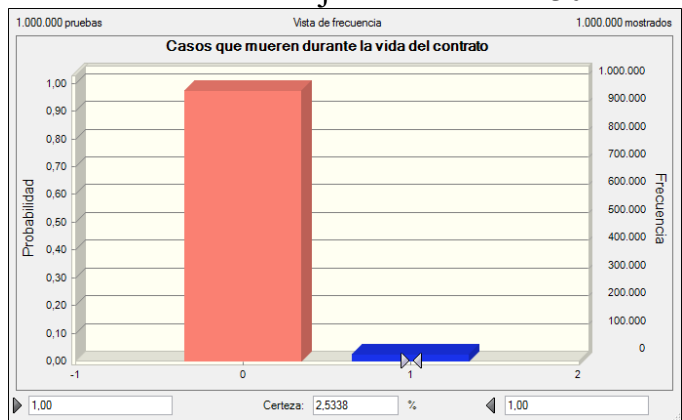

A.4. Probabilidad de ejercicio si edad $=40$

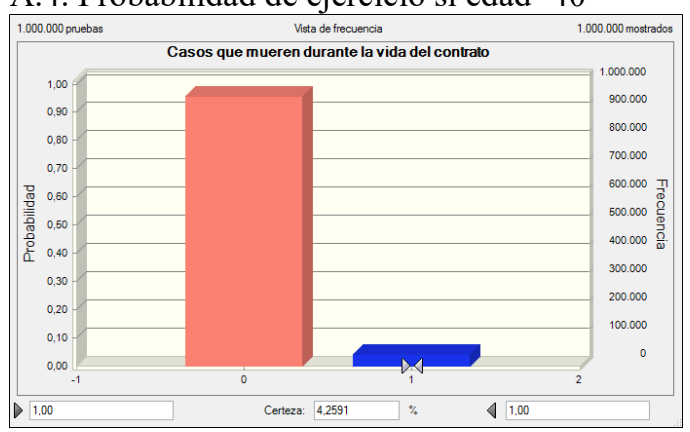

A.6. Probabilidad de ejercicio si edad $=50$

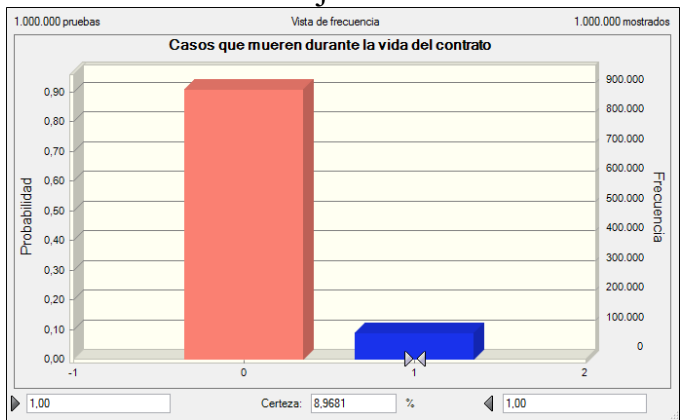

A.8. Probabilidad de ejercicio si edad $=60$

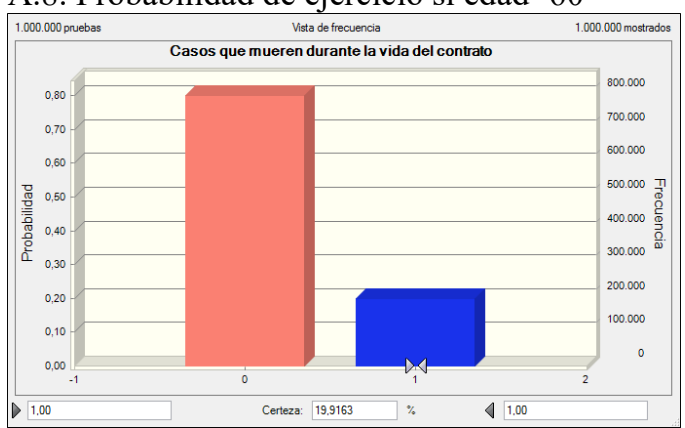




\section{A.9. Distribución si edad $=70$}

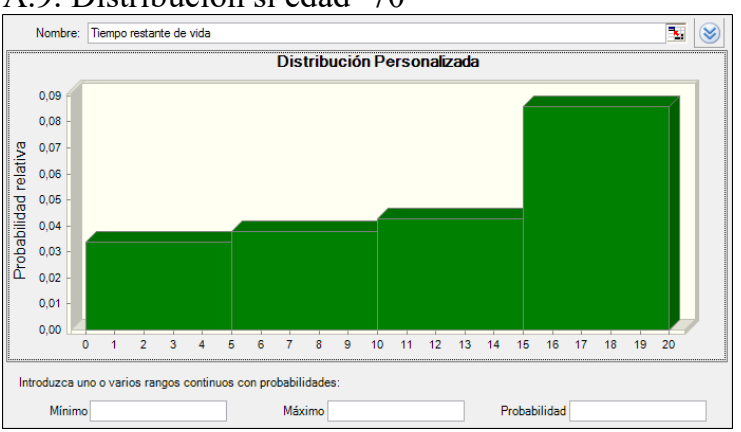

A.11. Distribución si edad $=40$ y género masculino

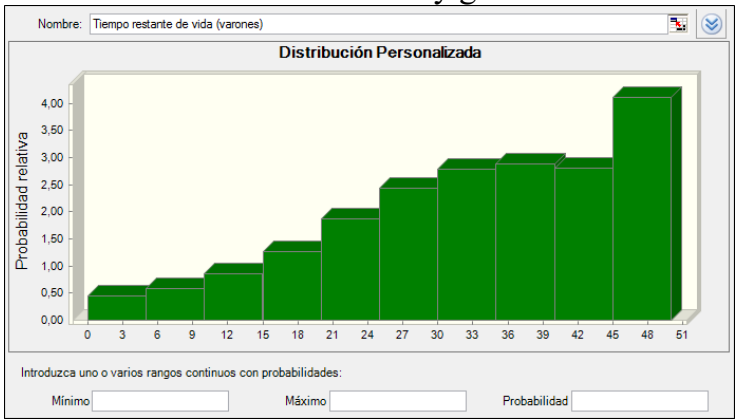

A.13. Distribución si edad $=40$ y género femenino

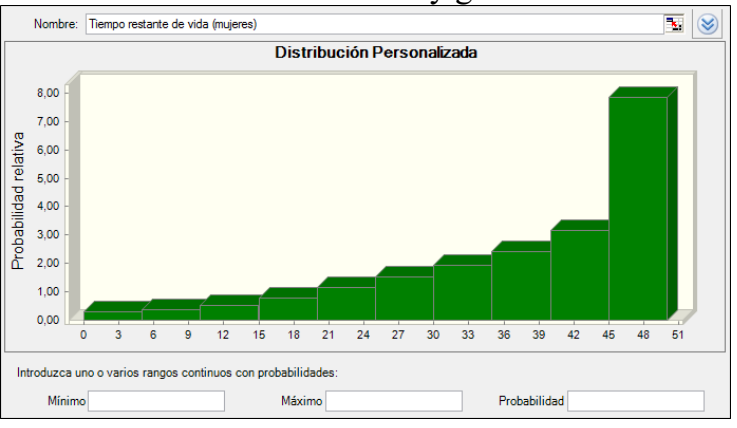

Fuente: Elaboración propia utilizando software Crystal Ball en Microsoft Excel ${ }^{\circledR}$.
A.14. Probabilidad de ejercicio si edad $=40$ y género femenino

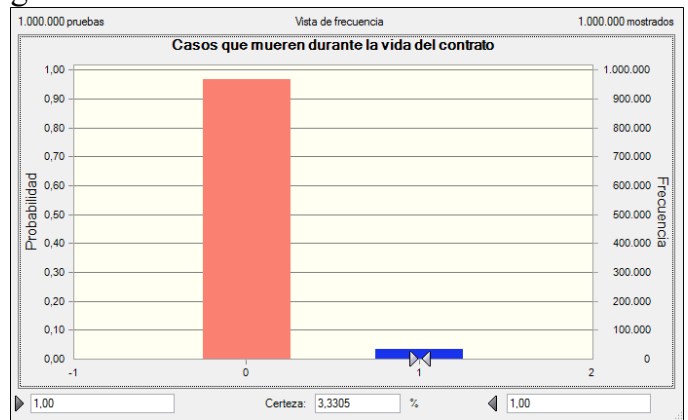

A.10. Probabilidad de ejercicio si edad $=70$

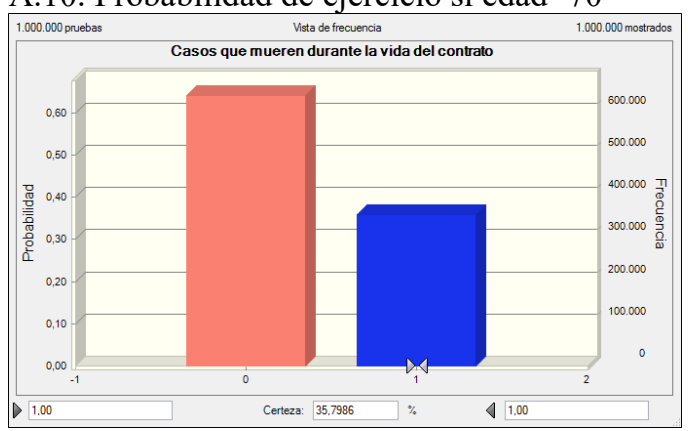

A.12. Probabilidad de ejercicio si edad $=40$ y género masculino

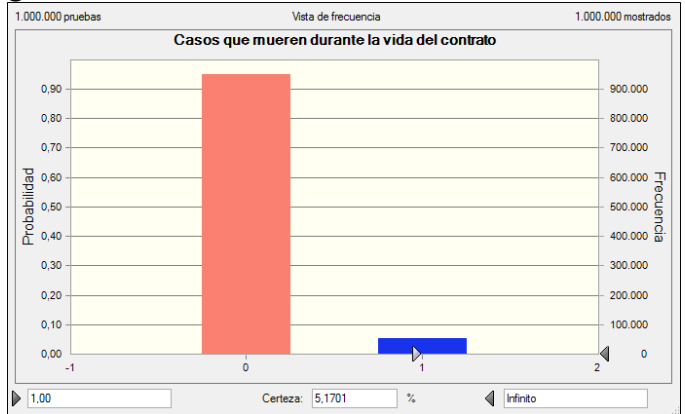

